\title{
Resting-State Functional Networks of Different Topographic Representations in the Somatosensory Cortex of Macaque Monkeys and Humans
}

\author{
John Thomas, Dixit Sharma ${ }^{1}$, Sounak Mohanta ${ }^{2}$ and Neeraj Jain* \\ National Brain Research Centre, Manesar 122052, India
}

\section{*Address correspondence to:}

Neeraj Jain

National Brain Research Centre

NH 8

Manesar, Haryana - 122052, India

Email: neeraj.jain@nbrc.ac.in; njain.nbrc@gmail.com

Phone: +91(124) 2845207; (+91) 9810446444

${ }^{1}$ DS current address: Behavioral and Neural Science Program, Rutgers University, Newark, USA

${ }^{2}$ SM current address: Dept of Psychology, University of Wisconsin - Madison, Madison, WI, USA 


\begin{abstract}
Information processing in the brain is mediated through a complex functional network architecture whose comprising nodes integrate and segregate themselves on different timescales. To gain an understanding of the network function it is imperative to identify and understand the network structure with respect to the underlying anatomical connectivity and the topographic organization. Here we show that the previously described resting-state network for the somatosensory area $3 \mathrm{~b}$ comprises of distinct networks that are characteristic for different topographic representations. Seed-based resting-state functional connectivity analysis in macaque monkeys and humans using BOLD- fMRI signals from the face, the hand and rest of the medial somatosensory representations of area $3 \mathrm{~b}$ revealed different correlation patterns. Both monkeys and humans have many similarities in the connectivity networks, although the networks are more complex in humans with many more nodes. In both the species face area network has the highest ipsilateral and contralateral connectivity, which included areas $3 \mathrm{~b}$ and 4 , and ventral premotor area. The area $3 \mathrm{~b}$ hand network included ipsilateral hand representation in area 4.
\end{abstract}

The emergent functional network structures largely reflect the known anatomical connectivity. Our results show that different body part representations in area $3 \mathrm{~b}$ have independent functional networks perhaps reflecting differences in the behavioral use of different body parts. The results also show that large cortical areas if considered together, do not give a complete and accurate picture of the network architecture.

\title{
Keywords
}

BOLD fMRI correlations; Resting-state connectivity; Area 3b; Somatotopy 


\section{Highlights}

- Somatosensory resting-state functional network is not uniform across the entire area $3 b$. Different body part representations have different connectivity networks.

- These functional connectivity networks have many similarities in the two primate species, i.e. macaque monkeys and humans, although the human network is more complex.

- In both the species network of the face representation is most extensive, which includes ipsilateral face motor cortex and PMv in both hemispheres.

- The hand representation in area $3 \mathrm{~b}$ has connectivity with ipsilateral hand motor cortex.

- Bilateral connectivity with homologous and nonhomologous area $3 b$ representations was observed only in humans.

- The functional connectivity networks largely reflect the underlying anatomical connectivity. 


\section{INTRODUCTION}

Since early descriptions of the cortical areas based on cytoarchitecture (Brodmann, 1909), various anatomical, neurophysiological and neuroimaging studies have given new insights into functional segregation across brain regions (see Amunts and Zilles, 2015). Cortical parcellation combining multiple features viz. cyto-, myelo- and immuno-architecture, topography, function and connectivity analysis help better define areal and sub-areal boundaries in the cortex and understand information processing networks (Glasser et al., 2016; Jain et al., 1998; Jain et al., 1994; Kuehn et al., 2017; Preuss et al., 1997; Van Essen et al., 2012). More recently, functional connectivity, particularly the correlation of intrinsic blood oxygenation level dependent (BOLD) fMRI signals from different brain areas, which is considered to represent functional association between regions, has been utilized for parcellation of the cortex (Gordon et al., 2016).

Resting-state functional connectivity is determined from correlations in the low frequency $(<0.01 \mathrm{~Hz})$ fluctuations of BOLD fMRI signals between different regions in a resting brain revealing functionally related areas (Biswal et al., 1995). Such time series cross-correlation analysis between a seed region of interest (ROI) and voxels in rest of the brain reveals functional connections across different brain regions even without any explicit task paradigm (Biswal et al., 1995). The correlated sets of voxels give rise to functional 'maps', also referred to as functional connectivity networks. These have been found to be consistent within and between subjects (Choe et al., 2015; Laumann et al., 2015; Shehzad et al., 2009). The resting-state functional connectivity has been studied in a variety of species ranging from mice, ferrets, cats, and monkeys to humans (Hutchison et al., 2013; Stafford et al., 2014; Zhou et al., 2016). Seed-based resting-state fMRI (rsfMRI) analysis enables tailored investigation to delineate networks spanning the entire brain. Furthermore, subject specific ROI analysis using rsfMRI data can reveal regional differences within a cortical area, as well as individual variations 
across different subjects (Braga and Buckner, 2017).

In functional connectivity studies traditionally somatosensory and motor cortical areas have been lumped together (Beckmann et al., 2005; Hutchison et al., 2011; Mantini et al., 2013). This network includes different topographic representations in the primary somatosensory, motor and secondary somatosensory areas (Disbrow et al., 2000; Manger et al., 1996; Nelson et al., 1980; Stepniewska et al., 1993). However, it is known from anatomical connectivity studies that different body part representations form independent information processing modules. For example, the hand and face representations in the somatosensory cortex have different interconnectivity, sources of feedback connections and interhemispherical connections (Chand and Jain, 2015; Fang et al., 2002; Killackey et al., 1983; Liao et al., 2013). Callosal connectivity also varies widely for different body part representations, for example, the face and trunk representations have higher connectivity as compared to the hand and foot regions (Killackey et al., 1983).

Resting-state connectivity largely reflects direct anatomical connectivity between different brain regions (Damoiseaux and Greicius, 2009; van den Heuvel et al., 2009; Wang et al., 2013), as well as functional connectivity between areas not directly connected anatomically (Adachi et al., 2012; Vincent et al., 2007). Recent neuroimaging studies have considered differences in cortical function and functional connectivity to delineate different brain areas (Glasser et al., 2016; Gordon et al., 2016; Sohn et al., 2012). Functional connectivity studies have found topography dependent network subdivisions within the somatomotor network in humans (Power et al., 2011; Yeo et al., 2011). Utilizing in vivo cortical myelin mapping and resting-state analysis, Kuehn et al. (2017) showed that the functional connectivity patterns in humans also follow the architectonic differences reiterating the importance of body part representations as an organizing principle for functional networks. 
We hypothesized that different body part representations within the primary somatosensory area $3 \mathrm{~b}$ have different connectivity networks with different functionally correlating nodes. In order to test this hypothesis, we analyzed seedbased resting-state fMRI connectivity of different body part representations. Further, to determine if the correlated features of these networks are evolutionarily conserved, we compared functional connectivity profiles in humans and macaque monkeys.

\section{METHODS}

\subsection{Subjects}

Five adult male macaque monkeys (Macaca mulatta), 8-11 years of age and weighing between 8-10 kg were used. All animal procedures were approved by the Institutional Animal Ethics Committee of National Brain Research Centre, and the Committee for the Purpose of Control and Supervision of Experiments on Animals (CPCSEA), Government of India, and conformed to NIH guidelines for care and use of animals in biomedical research. Twenty three right-handed human subjects (10 females and 13 males) between the ages of 22-39 years took part in the study. Participants had no history of neurological or psychiatric illness (selfreported). Informed consent was obtained from all the human subjects. Human study protocols were approved by the Institutional Human Ethics Committee.

\subsection{Data acquisition}

\subsubsection{Macaque monkeys}

For magnetic resonance data acquisition, macaque monkeys were initially anesthetized with ketamine hydrochloride ( $8 \mathrm{mg} / \mathrm{kg}$, IM). Glycopyrrolate $(0.015$ $\mathrm{mg} / \mathrm{kg}, \mathrm{IM}$ ) was administered in order to reduce the salivary secretions. When the 
monkeys were areflexive, they were intubated with an appropriately sized endotracheal tube and the anesthesia was switched to isoflurane (1-2\% in oxygen, Surgivet CDS 2000). T1 weighted anatomical scans were acquired using 1-2\% isoflurane in oxygen while the resting-state scans were taken using a lower isoflurane percentage $(0.5-0.8 \%$ in oxygen).

MR scans were acquired by transmitting radio-frequency pulses from a quadrature body-coil inside a 3-T clinical MRI scanner (Philips Achieva, Netherlands), and receiving signals using an eight-channel phased array knee coil (MRI Device Corporation, WI, USA) and sensitivity-encoding (SENSE) parallel acquisition (Pruessmann et al., 1999). Human knee coil was used for monkeys due to a better filling factor, which improved signal-to-noise ratio and the image quality (Dutta et al., 2014). Anesthetized animals were placed in supine position inside the scanner with the head completely inside the receiver coil. Head movements inside the coil were minimized by padding the gaps between the head and the coil with polyethylene foam blocks. T1 weighted anatomical scans of the brain were acquired using a 3D multishot Turbo Field Echo sequence (TR = 8.8 $\mathrm{ms} ; \mathrm{TE}=4.4 \mathrm{~ms}$; flip angle $=8^{\circ} ; 0.5 \mathrm{~mm} \times 0.5 \mathrm{~mm} \times 0.5 \mathrm{~mm}$ resolution; $250 \mathrm{~mm}$ x 210 mm x 56 mm field of view; 500 x 360 matrix; 112 transverse slices).

During the scans, physiological condition of the monkeys was continuously monitored with a MRI-compatible pulse-oximeter (Nonin 8600FO, USA), keeping constant isoflurane (0.5-0.8\%) and the oxygen flow rate $(1.5 \mathrm{l} / \mathrm{min})$. On each scanning day, functional data acquisition was always preceded by reference and anatomical scans. This enabled similar time lag between induction of anesthesia and the start of the functional scans across different days of acquisition.

2.2.1.1 Resting-state scans: Resting-state fMRI scans were acquired in oblique horizontal slice orientations, using a single-shot gradient echo echo-planar 
imaging $\left(\mathrm{TR}=2800 \mathrm{~ms} ; \mathrm{TE}=30 \mathrm{~ms}\right.$; flip angle $=90^{\circ} ; 1.5 \mathrm{~mm} \times 1.5 \mathrm{~mm}$ in-plane resolution; $2 \mathrm{~mm}$ thick slices; $96 \mathrm{~mm}$ x $102 \mathrm{~mm}$ x $54 \mathrm{~mm}$ field of view; 64 x 64 matrix; number of excitations $(\mathrm{NEX})=2$. Each resting-state scan session consisted of 140 functional volumes acquired over approximately 13 minutes.

From the five monkeys, a total of 32 scans were acquired. The number of sessions for different monkeys were 11,8, 6, 4 and 3, which were acquired on different days.

2.2.1.2. Functional localiser scans. For the functional location studies, the scanning parameters were similar to an earlier study from our lab (Dutta et al., 2014). Macaque monkeys were initially anesthetized using ketamine $(8 \mathrm{mg} / \mathrm{kg}$ IM) and xylazine ( $0.4 \mathrm{mg} / \mathrm{kg}$ IM). Subsequent maintenance of anaesthetic depth as required during scanning was achieved using low doses of ketamine (1.5 $\mathrm{mg} / \mathrm{kg} \mathrm{IM}$ ). Stimulation of the glabrous skin of the digits and palmar surface was done manually using a polyester brush at a frequency of approximately $2 \mathrm{~Hz}$. Cutaneous stimulation to chin was delivered using a smaller brush at the same frequency (see Dutta et al., 2014, Jain et al 1997, 2008). The stimulation frequency was paced by steady counting with respect to a clock, and was always done by the same experimenter. The functional MRI scans were acquired using the same MRI scanner (3T, Philips Achieva, Netherlands) with oblique horizontal slice orientations, using a single-shot gradient echo echo-planar imaging (TR = $2800 \mathrm{~ms} ; \mathrm{TE}=30 \mathrm{~ms}$; flip angle $=90^{\circ} ; 1.5 \mathrm{~mm} \times 1.5 \mathrm{~mm}$ in-plane resolution; 2 $\mathrm{mm}$ thick slices; NEX = 2; $96 \mathrm{~mm} \times 102 \mathrm{~mm}$ x $54 \mathrm{~mm}$ field of view; 64 x 64 matrix). Acquisition paradigm using standard block design consisted of alternating rest (28s) and stimulation (28s) blocks. During the scans physiological condition of the monkeys was continuously monitored with a MRI-compatible pulse-oximeter (Nonin 8600FO, USA). In each session 140 functional volumes were acquired. 


\subsubsection{Humans}

Data from human subjects were acquired in the same 3-T MRI scanner but using an 8 channel SENSE head coil. Anatomical T1 weighted images were acquired for each subject using 3D multishot TFE sequence $\left(\mathrm{TR}=8.4 \mathrm{~ms}\right.$; flip angle $=8^{\circ}$; FOV $=250 \mathrm{~mm} \times 230 \mathrm{~mm} \times 150 \mathrm{~mm} ; 252 \times 211$ matrix; 150 slices) before the functional scanning sessions.

2.2.2.1 Resting-state scans. Resting-state functional scans were acquired with a $\mathrm{T}_{2} *$ weighted gradient echo EPI sequence $(\mathrm{TR}=2000 \mathrm{~ms} ; \mathrm{TE}=30 \mathrm{~ms}$; flip angle $=70^{\circ} ; 3 \mathrm{~mm} \times 3 \mathrm{~mm}$ in-plane resolution; slice thickness $4 \mathrm{~mm}$; FOV $=230 \mathrm{~mm} \times$ $242 \mathrm{~mm}$ x $132 \mathrm{~mm} ; 33$ slices). Two hundred functional volumes were acquired during which the participants lay in supine position with palms facing downwards. They were instructed not to intentionally move any body part, keep their eyes closed and try not to indulge in any active thought process. Data was acquired in two successive sessions on a single day from each subject.

Of the 23 subjects, the data from two subjects (both females) were discarded due to excessive head motion and self-reported sleepiness during the resting scans. Data from additional four subjects ( 2 females, 2 males) were not considered due to involuntary hand movements during the acquisition. Data acquired in 34 scans from the remaining 17 subjects (6 females, 11 males) were used for further analyses.

2.2.2.2. Functional localiser scans. For acquiring BOLD signals in response to peripheral stimulation in humans, the chin and the glabrous skin of the digits and palmar surface of the hand were stimulated manually using a polyester brush at a frequency of $2 \mathrm{~Hz}$ as for the monkey stimulation. The functional MRI scans were 
acquired using a single-shot gradient echo echo-planar imaging (TR = 2000 ms; $\mathrm{TE}=30 \mathrm{~ms}$; flip angle $=70^{\circ} ; 3 \mathrm{~mm}$ x $3 \mathrm{~mm}$ in plane resolution; slice thickness 4 mm; FOV = 230 mm x 242 mm x 132 mm; 33 slices). Two hundred volumes were acquired per session using standard block design consisting of alternating rest and stimulation blocks. The functional scans were not acquired on the day of the resting-state scans.

\subsection{Pre-processing}

Monkey and human data were processed using statistical parametric mapping (SPM8) software (http://www.fil.ion.ucl.ac.uk/spm/software/spm8) operating in Matlab 2013a platform (MathWorks Inc., MA, USA). The acquired structural images were aligned in the anterior commissure-posterior commissure (AC-PC) plane with coordinates of AC set to zero. After removal of the dummy scans, the functional images were slice time corrected to remove time lag between slices within a volume. These slice-time corrected functional volumes were head motion corrected using a six-parameter affine 'rigid body' transformation to minimize differences between each successive scan and the reference scan (the first scan in the time series). Motion corrected functional volumes of monkeys were co-registered with the corresponding high-resolution subject specific structural images. For humans, structural images were normalized to the standard template (ICBM 2009a Nonlinear Symmetric template; Fonov et al., 2009). Images were then visually inspected to check the registration.

For seed to voxel analysis (see below) data were smoothed with a $2 \mathrm{~mm}$ (macaques) or $8 \mathrm{~mm}$ (humans) Full Width at Half Maximum Gaussian kernels. For ROI-ROI analysis unsmoothed data was used to avoid possible spill over from the neighboring voxels due to large smoothing kernels.

Functional connectivity for both macaques and humans was determined using 
CONN toolbox (version 15.e) for SPM (Whitfield-Gabrieli and Nieto- Castanon, 2012). Nuisance covariates of cerebrospinal fluid and white-matter signals were modeled and removed following CompCor strategy (Behzadi et al., 2007), as implemented in CONN. Linear regression was performed where signals from the white matter and the cerebrospinal fluid, along with the global signal and the motion parameters were taken as covariates of no interest. This was followed by a temporal band pass filtering $(0.008-0.09 \mathrm{~Hz})$ to reduce low frequency drifts and the high frequency physiological noise. Before calculating bivariate correlation coefficients across brain regions, the processed data were despiked with hyperbolic tangent squash function and linearly detrended to remove low drift scanner noise.

For analysis of the activation fMRI data in monkeys, subject specific analysis was done as described before (Dutta et al., 2014). The stimulation epoch was represented using a box car model which was convolved with a haemodynamic response function (hrf) as implemented in SPM. For humans, the analysis was done in ICBM template space using normalized functional images. Motion estimates were included in the regression model as covariates of no interest. General linear model based univariate analysis yielded statistical parametric maps which were thresholded using uncorrected $p<0.005$ for monkeys as reported earlier (Dutta et al., 2014). The cluster selection criterion was set to two or more contiguous voxels (see Dutta et al., 2014). In humans the activation clusters were thresholded using family wise error correction (FWER) at $p<0.05$. Statistical maps were overlaid on the standard template images after transforming to the INIA19 standard space in monkeys and ICBM template in humans for comparative visualization (see Fig. 2).

\subsection{Regions of Interest (ROIs)}

For determining resting-state functional connectivity of different somatosensory 
representations, we demarcated ROI's for area 3b, and the face representation (face $3 b$ ), hand representation (hand $3 b$ ), and rest of the medial region (med3b) in area $3 \mathrm{~b}$ (Fig. 1). Other regions of interest (ROIs) for both monkeys (MK) and humans (HU) were demarcated as described below. The ROI's were drawn on successive parasagittal slices and confirmed by reexamining the ROI's in coronal and axial planes (Fig. 3).
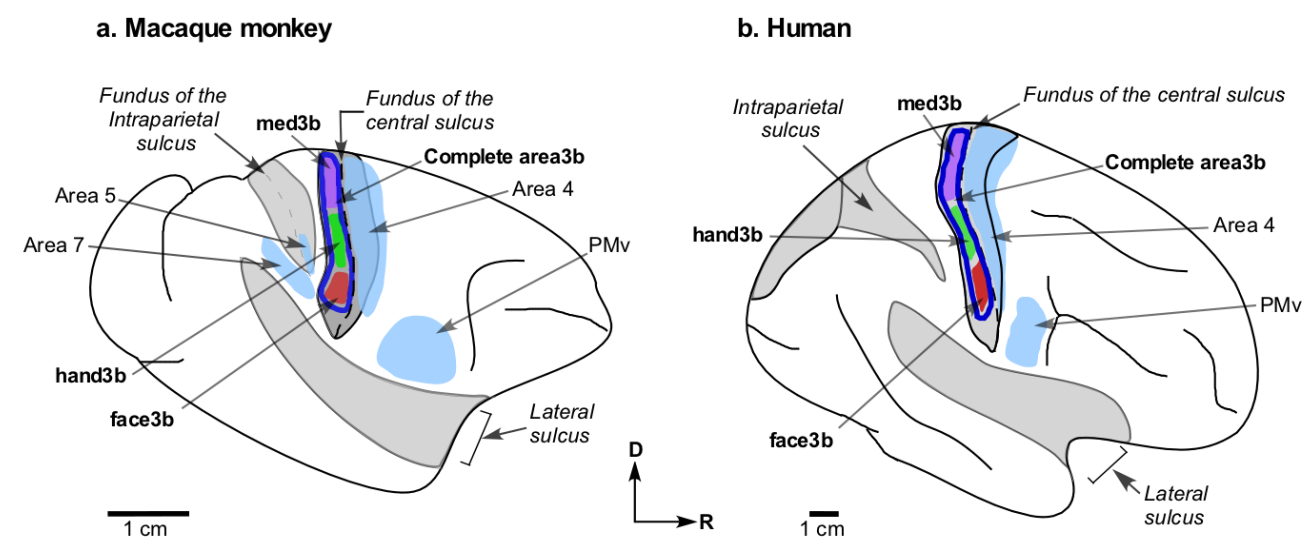

Fig. 1. Schematic representation of the regions of interest (ROI's) used for the seed-based resting-state functional connectivity analysis of the somatosensory cortex in (a) macaque monkeys and (b) humans. Complete area 3b ROI (thick dark blue outline; area3b), and ROls of different body part representations - face3b (red), hand3b (green) and med3b (violet) are shown. The target ROl's are light blue. The ROl's are shown on outline drawings of lateral view of the cortical surface. The major sulci are labelled in italics for reference. Some of the sulci are shown opened (grey shading). S2 ROI is not visible in this view. PMv, Premotor Ventral Area; D, dorsal; R, rostral.

\subsubsection{Macaque monkeys}

The ROI were drawn (Fig. 1a) on subject specific high resolution T1 images in both the hemispheres. The entire medio-lateral extent of the primary somatosensory area $3 \mathrm{~b}$ on the anterior bank of the post central gyrus, excluding the medial wall representations was taken as the complete area $3 \mathrm{~b}$ seed ROI (area3b). The ROI was restricted to grey matter using a grey matter mask. The boundaries between area $3 \mathrm{~b}$ and $3 \mathrm{a}$, and area $3 \mathrm{~b}$ and 1 were demarcated with reference to the cytoarchitectonic and electrophysiology studies in monkeys (Chand and Jain, 2015; Jain et al., 2008; Nelson et al., 1980; Tandon et al., 2009). 
From these data area $3 \mathrm{~b}$ was judged to lie between the depths of $1.5 \mathrm{~mm}-8 \mathrm{~mm}$ from the lip of the central sulcus (Fig. 3). To ensure that area $3 b$ does not extend into the adjacent areas, voxels adjacent to the rostral boundary with area $3 \mathrm{a}$ and caudal boundary with area 1 were scrubbed off.

The ROI's for body part representations (Fig. 3) were drawn within limits of area $3 \mathrm{~b}$ boundaries with reference to the published electrophysiological and anatomical studies, and monkey atlases (Chand and Jain, 2015; Jain et al., 2008; Nelson et al., 1980; Paxinos et al., 2000; Saleem and Logothetis, 2006). A perpendicular from the tip of the intraparietal sulcus to the central sulcus on the surface of the brain was considered as the hand-face border, i.e. the medial limiting boundary of the face ROI (Chand and Jain, 2015; Jain et al., 2008; Manger et al., 1997). The face ROI included the contralateral representations of face regions and excluded the antero-lateral representations of ipsilateral trigeminal and intraoral inputs (Manger et al., 1996). The hand ROI was restricted to $7 \mathrm{~mm}$ distance medial from the hand-face border (Jain et al., 2008; Kambi et al., 2011, 2014; Nelson et al., 1980; Tandon et al, 2009). This included the hand representation i.e., digits and palm, but excluded other forelimb representations. Accuracy of the hand and chin ROI's was further confirmed by referencing the fMRI activation loci in response to the chin and the hand stimulation in the same monkeys (Fig. 2a; also see Dutta et al., 2014).

The remaining medial region of area $3 \mathrm{~b}$ excluding the medial wall was considered together as the third ROI termed med3b. This included representations of the shoulder, trunk, foot and parts of the leg that are on the dorsal surface (Jain et al., 2008; Nelson et al., 1980). This ROI also included medial-most parts of the upper arm representation. Due to the uncertainties in demarcating boundaries between these representations, the combined ROI was used. Care was taken to avoid voxel overlap between face $3 b$, hand $3 b$, and med3b ROIs in the medio-lateral direction as well by having adequate gap between the representations by scrubbing off 
voxels.

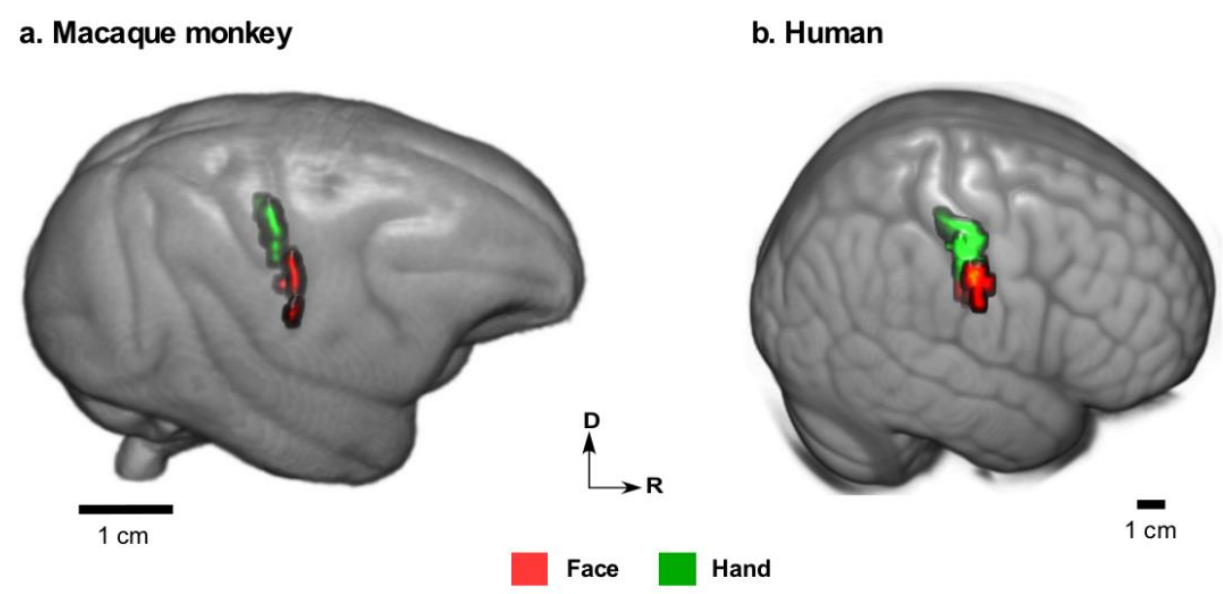

Fig. 2. Voxels activated in fMRI scans of (a) macaque monkeys $(n=5)$ and (b) humans $(n=$ 5) when the hand, i.e. glabrous digits and palm (green) or the face (red) was undergoing tactile stimulation. The voxels with peak activation in the post central gyrus are rendered on respective standard brain template image. All other brain regions are masked out. Statistical thresholds are $p<0.005$ (uncorrected) for monkeys and $p<0.05$ (FWER corrected) for humans. D, dorsal; R, rostral.

Other ROIs, the primary motor cortex (area4), ventral premotor cortex (PMv) and secondary somatosensory cortex (S2) were drawn with reference to the published anatomical and electrophysiological data, and macaque brain atlases (Barbas and Pandya, 1987; Burton et al., 1995; Krubitzer et al., 1995; Paxinos et al., 2000; Saleem and Logothetis, 2006). ROIs were drawn for the body part representations in area 4 to demarcate the face (face4), the hand (hand4) and the remaining medial region (med4) in accordance with the published reports (Stepniewska et al., 1993).

\subsubsection{Humans}

The ROIs were delineated on the standard brain template (Fonov et al., 2009). The complete area $3 b$ ROI (area3b) included the deeply situated area $3 b$ on the posterior bank of the central sulcus extending from the lateral face area to medial boundary where the central sulcus meets the midline sulcus (Blankenburg et al., 2003). This ROI excluded the medial wall representations of area 3b (Fig. 1b). 
bioRxiv preprint doi: https://doi.org/10.1101/775569; this version posted October 6, 2020. The copyright holder for this preprint (which was

not certified by peer review) is the author/funder, who has granted bioRxiv a license to display the preprint in perpetuity. It is made available under aCC-BY-NC-ND 4.0 International license.

\section{Lateral boundary of area $3 \mathrm{~b}$ extended upto the lateral boundary of the face ROI}

(see below). The voxels included in the area 3b ROI was confined within the grey

\section{a. Macaque monkey}
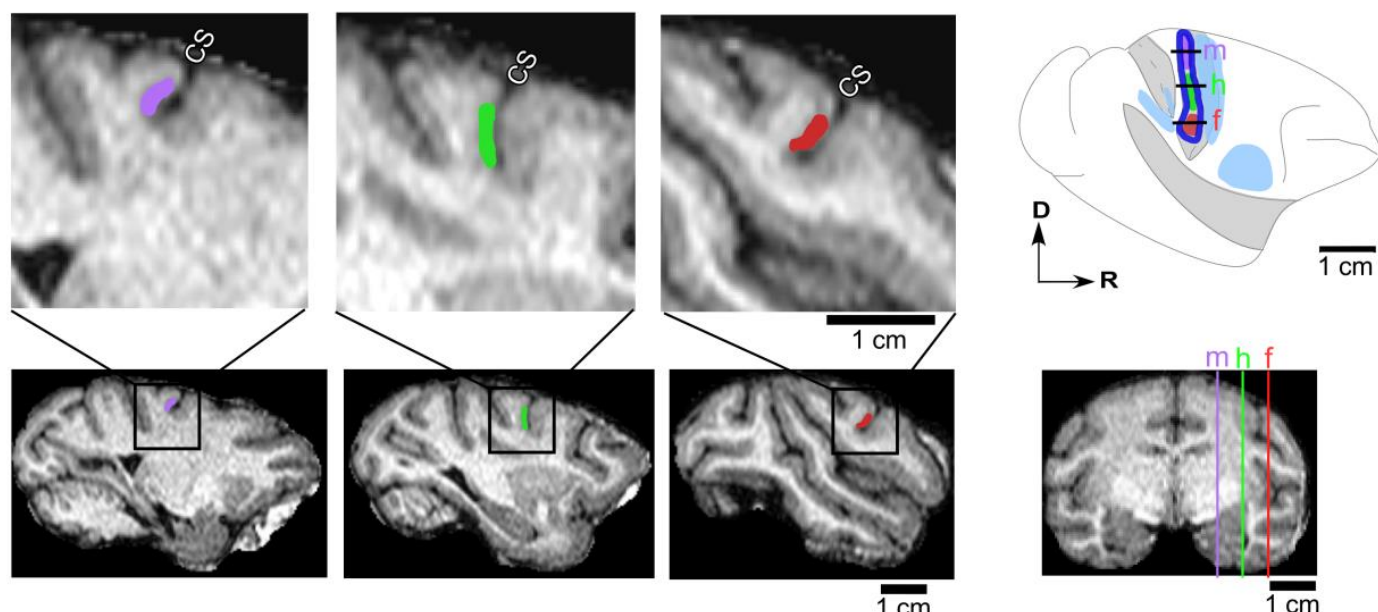

b. Human

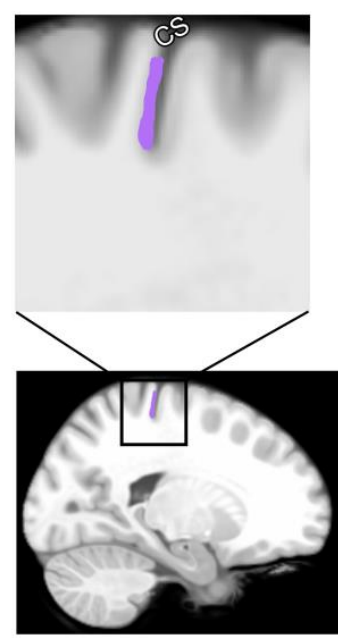

D

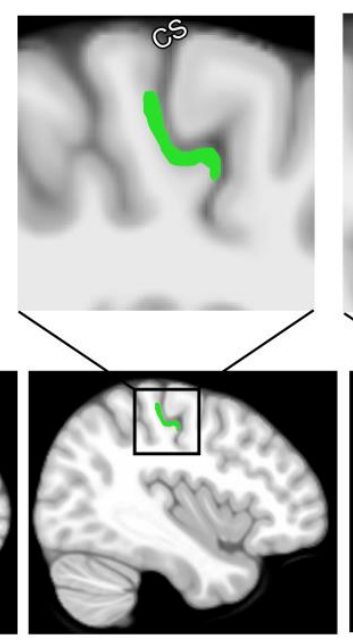

hand3b

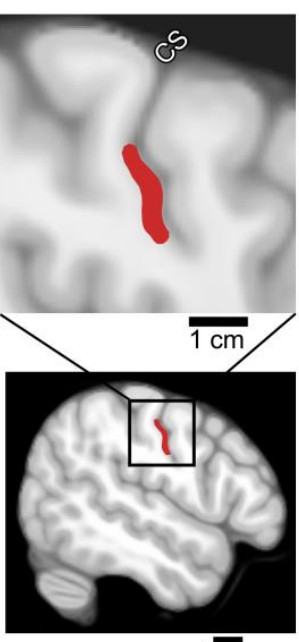

$1 \overline{c m}$
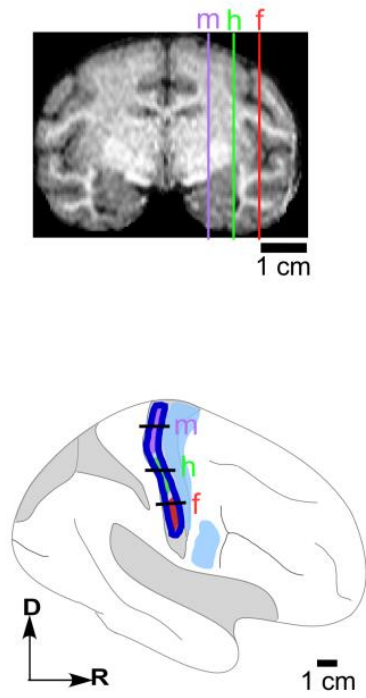

$1 \overline{\mathrm{cm}}$

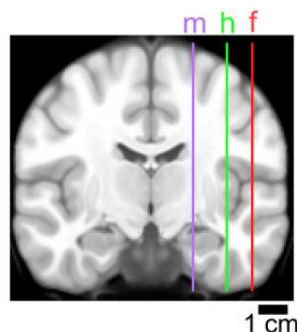

Fig. 3. Examples showing how region of interests (ROl's) used for functional connectivity analysis were drawn in area $3 \mathrm{~b}$ of (a) macaque monkeys and (b) humans. The ROl's were drawn on the subject specific high resolution T1 structural image in monkey and template brain in humans. Upper row on the left in 'a' and 'b' show ROl's for the face (red), hand (green) and medial representations (violet) marked on representative sagittal slices shown enlarged in the region of the central sulcus (CS). Second rows in 'a' and 'b' on the left show complete sagittal slices with boxes marking the region shown enlarged in the upper rows. Upper right in 'a' and 'b' show locations of the slices marked by horizontal black lines on drawings of the dorsolateral surface of the brains to show their position with respect to the CS. Locations of slices are also shown as color coded lines on the coronal slices of the brains in the lower right ( $f$, face $3 b ; h$, hand3b; $m$, med3b). D, dorsal; $R$, rostral. 
matter. A grey matter mask was used to exclude white matter voxels. The boundaries between area $3 \mathrm{~b}$ and area 1 , area $3 \mathrm{~b}$ and area $3 \mathrm{a}$ were delineated with reference to published anatomical studies, SPM Anatomy toolbox and human brain atlas (Bakker et al., 2015; Eickhoff et al., 2005; Geyer et al., 1999, 2000b). Based on these rostral boundary of area $3 \mathrm{~b}$ was estimated to lie at depths varying from $7 \mathrm{~mm}$ from the lip of the central sulcus in the region of the trunk representation to $40 \mathrm{~mm}$ near the hand representation (Fig. 3).

The hand (hand3b) and the face (face3b) ROIs were then delineated. The anterior and posterior extents of the face $3 b$ and hand $3 b$ were drawn according to the published anatomical studies (Geyer et al., 1999, 2000b). The inverted $\Omega$ shaped knob was taken as suggestive of the motor hand area (Yousry et al., 1997). The somatosensory hand representation presumed to be lying in apposition to the area 4 hand region was drawn on the post central gyrus with the 'knob' as a guide. Other imaging studies were also used as a reference for location of the hand area (Blankenburg et al., 2003; Maldjian et al., 1999; Nakamura et al., 1998). Face ROI was drawn lateral to the hand ROI taking care to exclude the very lateral representations of intra-oral regions and tongue (Miyamoto et al., 2006). The lateral part of the face representation overlies the deeper medial part of the tongue representation, which was carefully excluded. We acquired fMRI data while stimulating the entire hand (i.e. glabrous digits and palm) or the chin, and used these data to confirm the placement of the ROI's (Fig. 2b).

Similar to monkeys, med3b in humans comprised of parts of area $3 \mathrm{~b}$ medial to the hand representation up to the location where central sulcus meets the midline, and excluded the medial wall representations. Voxels near the ROI edges in area $3 \mathrm{~b}$ were scrubbed off to avoid mediolateral overlap between them.

Other ROIs - area4, PMv and S2 were also drawn on the standard ICBM template. ROI's for area 4 (the primary motor cortex) and PMv (ventral premotor 
cortex) were drawn using SPM Anatomy toolbox with reference to the available anatomical data (Eickhoff et al., 2005; Geyer et al., 1996, 2000a, 2004).

Secondary somatosensory ROI, which included both S2 and PV, was drawn using SPM Anatomy toolbox and referring to the published literature on S2 topography (Blatow et al., 2007; Disbrow et al., 2000; Eickhoff et al., 2005, 2007; Ferretti et al., 2004).

ROIs were drawn for the body part sub-divisions of the primary motor cortex to demarcate the face (face4), the hand (hand4) and the remaining medial region (med4) in accordance with the published reports (Geyer et al., 2000a; Schieber, 2001).

\subsection{Connectivity analysis}

For both monkeys and humans, seed-to-voxel connectivity was determined using CONN toolbox. For analysis in each human subject, the two successive sessions, which were acquired on the same day were concatenated. We did preliminary analysis taking the first and the second sessions of all the subjects separately. However, since no significant differences was found between the two sessions (not shown), the sessions were concatenated. Each session of the monkeys, which were all acquired on separate days, was analyzed separately. Data from each ROI were averaged across the sessions for each animal. To determine connectivity maps complete area $3 b$, face $3 b$, hand $3 b$ and med3b ROI's were used as seed regions. While performing linear regression in $\mathrm{CONN}$, motion parameters were taken as regressors. Resultant beta maps, which were Fisher-z transformed correlation value maps, were used for further statistical analysis.

\subsubsection{Seed-to-voxel analysis}

To perform group analysis for monkeys, the subject level beta maps were 
transformed to standard INIA19 template space (Rohlfing et al., 2012) using transformation matrix. The transformation matrix was generated using FSL's linear and non-linear registration tool FLIRT and FNIRT respectively, registering subject specific high resolution T1 image to INIA19 template space (Jenkinson et al., 2002). Transformed beta maps in the template space were statistically tested using one sample t-test with null hypothesis of no correlation at $p<0.01$ with False Discovery Rate (FDR) correction. Resultant group level statistical maps were then mapped on inflated brain surface using CARET (ver. 5.616; Van Essen et al., 2001) and were displayed on slices using MRIcron software (ver. 1; Rorden and Brett, 2000).

For human subjects, subject level beta maps were used for the statistical group analysis performed using one sample t-test with null hypothesis of no correlation thresholded at $p<0.01$ with FDR correction. The group level statistical maps were mapped on inflated standard template brain surface using BrainNet Viewer (Xia et al., 2013) and were displayed on slices using MRIcron software (ver. 1; Rorden and Brett, 2000).

\subsubsection{ROI-ROI analysis}

To calculate the effect size of highly correlating ROIs, specific ROI-ROI Fisher-z transformed correlations were calculated between different ROI's using CONN. One sample t-test was performed at the subject level in both monkeys and humans with null hypothesis of no correlation with a threshold for significance set for monkeys at $p<0.05$, and humans at $p<0.00001$. The thresholds were decided empirically referring to the connectivity networks reported for the somatomotor cortex (Biswal et al., 1995; Kuehn et al., 2017; Mantini et al., 2013; Vincent et al., 2007; see 'limitations of the study' in 'Discussion'). Goal was to avoid spurious connectivity while ensuring that the known connectivity patterns were not thresholded out. 
To statistically compare the connectivity of ROI-ROI pairs, two-way ANOVA $($ alpha $=0.05)$ and post hoc Tukey test $(p<0.01)$ was implemented using GraphPad Prism 6.01 for Windows (GraphPad Software, California USA, Www.graphpad.com). All other ROI-ROI statistical tests were performed in MATLAB.

\section{RESULTS}

We determined resting-state functional connectivity networks of different topographic representations in area $3 \mathrm{~b}$ of macaque monkeys and humans. Exploratory seed-to-voxel correlation analysis was first done to reveal functionally connected regions in the brain. The networks thus revealed were further analyzed by ROI-ROI correlation analysis. We describe below our results for macaque monkeys and humans together for ease of comparison.

\subsection{Somatosensory resting-state networks: seed-to-voxel correlation analysis}

Resting-state network organization was determined taking the entire area $3 \mathrm{~b}$ or different body part representations as seeds for the exploratory correlation analysis. The networks were broadly similar for both humans and monkeys (Fig. 4 and 5).

The network of complete area $3 b$ (area3b) in both the primate species comprised of contralateral area $3 \mathrm{~b}$. In both the hemispheres area3b network also had nodes in area 4, second somatosensory area (S2), premotor ventral area (PMv) and insula (Fig. 5). In monkeys it also included area 7, putamen and area 5 of both the hemispheres (Fig. 5a).

Further analysis of connectivity of different body part representations revealed that each representation had a distinct connectivity pattern as described below. 
The face representation (face $3 b$ ), in both monkeys and humans showed strong connectivity with the face representation in contralateral area $3 b$. Face $3 b$ also showed bilateral connectivity with the face representation in area 4 (face4), PMv, S2, and insula (Fig. 4 and 5). However, only in monkeys, face $3 b$ showed bilateral connectivity with area 7 and putamen (Fig. 5a).

a. Macaque monkey

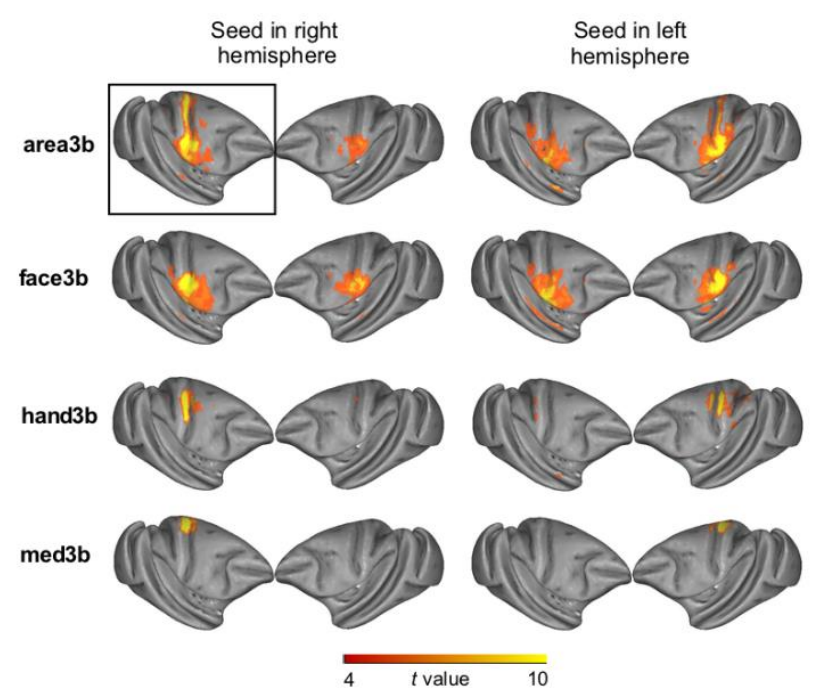

c.

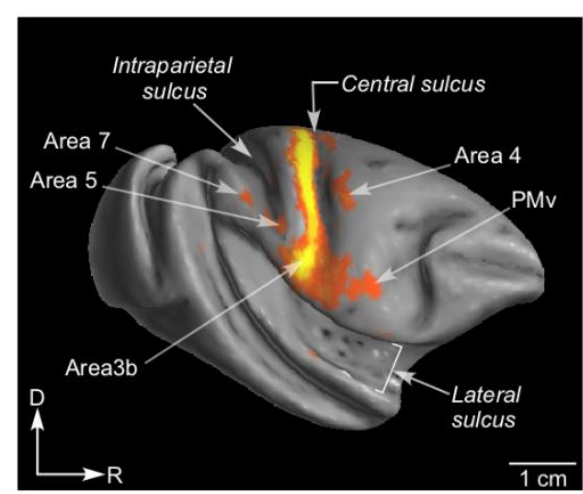

b. Human

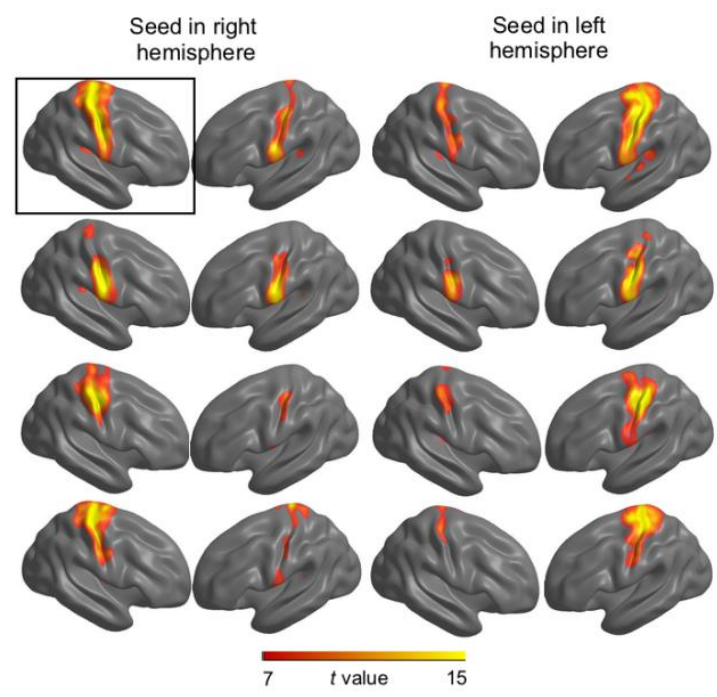

d.

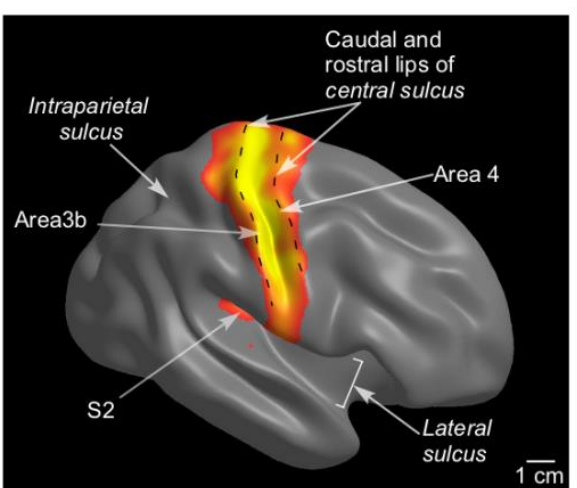

Fig 4. Seed to voxel resting-state functional connectivity of the somatosensory cortex of (a, c) macaque monkeys and (b, d) humans. (a and b) Bi-hemispherical views of the somatosensory network in macaques and humans shown on partially inflated cortical surfaces when the seed was complete area 3b (area3b; top row), face representation in area $3 \mathrm{~b}$ (face $3 \mathrm{~b}$; second row), the hand representation (hand3b; third row), or the medial part of area $3 b$ (med3b; bottom row). To illustrate symmetrical nature of the network, data are shown for seed in the left as well as the right hemisphere (see labels on top). The 't-values' correspond to statistical significance of $p<0.01$ (FDR corrected). Note that t-values are scaled to highlight the differences in connectivity maps of ROl's (see the colour scale bars at the bottom).' $c$ ' and 'd' show enlarged view of the boxed figurines in 'a' and 'b' respectively to illustrate the details. D, dorsal; R, rostral; orientation arrows shown in 'c' also applies to 'd'.

The other two ROIs i.e. hand $3 b$ and med $3 b$ revealed a network with fewer nodes 
than face $3 b$. In monkeys the hand $3 b$ network included area 5 and the hand representation in area 4 (hand4) of both the hemispheres. The human hand3b showed connectivity to the contralateral hand $3 b$ and the ipsilateral hand4 (Fig. 4 and 5). Med3b in both monkeys and humans showed connections to the rostrally adjacent medial part of area 4 (med4) and the contralateral med3b (Fig. 5).
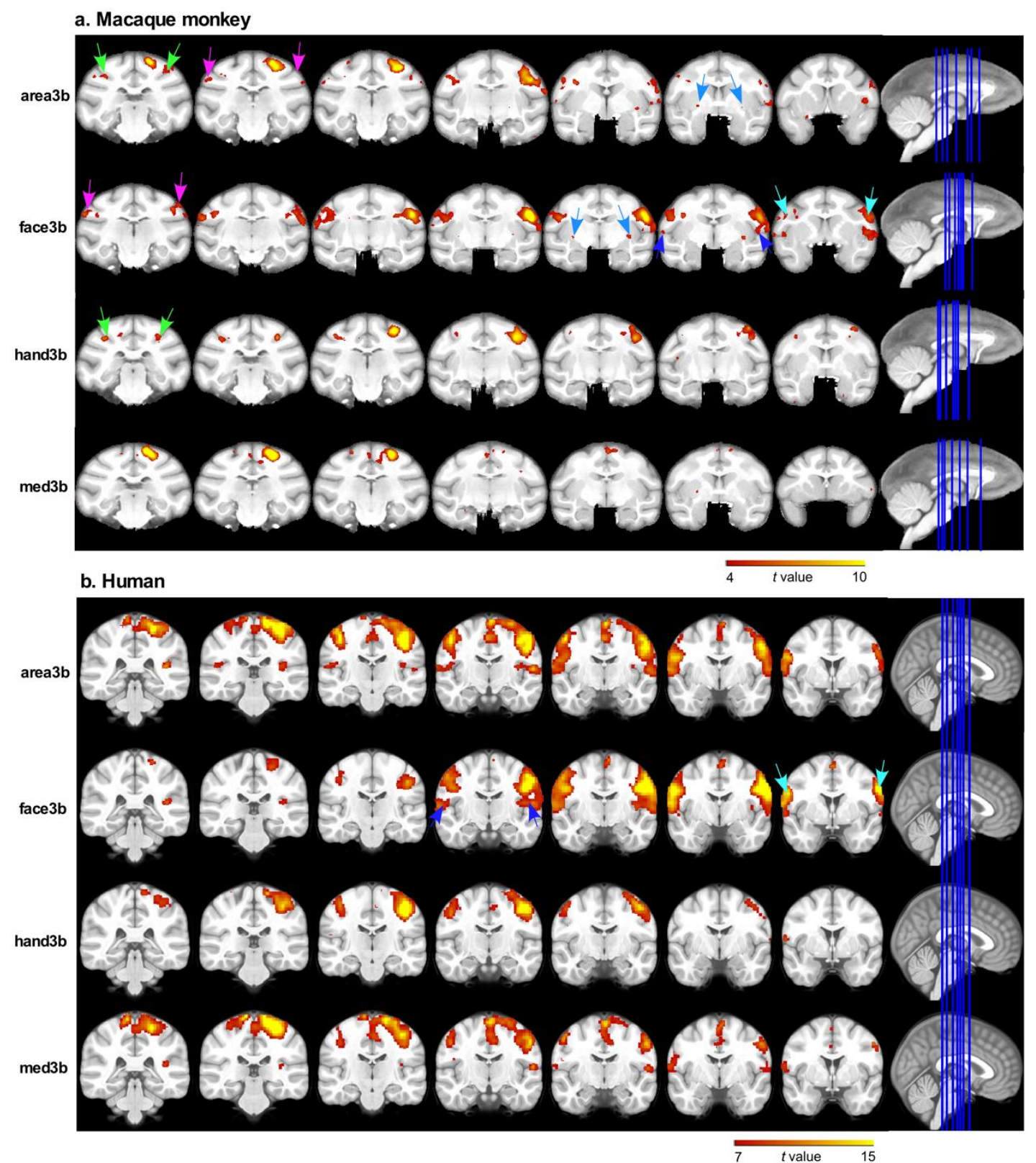

Fig. 5. Seed to voxel correlations in area $3 \mathrm{~b}$ of the right hemisphere of (a) monkeys and (b) humans shown on a series of coronal slices. The slices are arranged in the caudal to rostral order from left to right. The right-most column shows parasagittal sections of the brain with the blue lines marking the plane from which the coronal slices are taken. Correlations with area 7 (pink arrows), area 5 (green arrows) and putamen (blue arrows) were observed only in monkeys. Locations of S2 (violet arrows) and PMv (cyan arrows) are also marked. M, medial; R, rostral. Other conventions as for Figure 4. 
The results showed that the functional connectivity network of area $3 b$ is different for different body part representations. Furthermore, a seed ROI placed in either hemisphere had generally similar connectivity pattern, suggesting that the networks are largely laterality independent (Fig. 4).

The nodes of the network revealed by seed-to-voxel connectivity results guided the ROI-ROI analysis described below.

\subsection{ROI-ROI correlations: Different body part representations in area 3b contribute differentially to the complete area $3 \mathrm{~b}$ functional connectivity}

For a detailed investigation of the results of the seed-to-voxel analysis, ROI-ROI analysis was performed. ROIs were drawn in the seed as well as the target areas in the primary somatosensory and motor areas, S2 and PMv. In order to determine the extent of connectivity between correlated regions BOLD signals from all the voxels in each ROI were averaged and time series correlations of the averaged signals were determined for each ROI-ROI pair. Color coded connectivity matrices were constructed using averaged Fisher-z transformed correlation coefficients $\left(C C_{Z}\right)$ for these ROI pairs in both humans and macaque monkeys to illustrate the results (Fig. 6).

In both the species ROI encompassing entire area $3 \mathrm{~b}$ in either of the hemisphere revealed significant connections with the contralateral area $3 b$. Area $3 b$ in the right hemisphere also had significant bilateral correlations with area 4 (Fig. 6). In humans, area $3 \mathrm{~b}$ of right hemisphere also showed bilateral connectivity with S2 and PMv, whereas area $3 \mathrm{~b}$ of the left hemisphere has connectivity with only ipsilateral S2 and PMv. Area 3b of the left hemisphere in monkeys showed correlations with bilateral S2 and ipsilateral area 4 (Fig. 6).

Looking at the connectivity of individual body part representations, in both the 
species, face $3 \mathrm{~b}$ in the right hemisphere showed significant bilateral connectivity with face representation in area 4 and PMv. In monkeys face $3 b$ of the right hemisphere had significant connectivity with ipsilateral S2, and face $3 \mathrm{~b}$ of the left hemisphere with contralateral S2. This essentially means that right S2 had connectivity with face $3 b$ in both the hemispheres. In humans, face $3 b$ had significant connections with the contralateral face $3 \mathrm{~b}$, and bilateral connectivity with S2 (Fig. 6).

The hand $3 b$ in both species had significant connectivity with ipsilateral hand4. Med3b in both hemispheres of humans and the left hemisphere of monkeys had significant correlations to homotopic representation in area 4 (one sample t-test, for $p$-values see Tables 1 and 2).

Thus ROI-ROI analysis revealed that face $3 \mathrm{~b}$ has more widespread and complex functional network than med $3 b$ and hand $3 b$. The network of complete area $3 b$ ROI largely includes networks of all the three representations viz. face $3 b$, hand $3 b$ and med3b, although some of the nodes are seen only when individual body parts are considered separately. For example, in monkeys bilateral connectivity with PMv, and in humans bilateral connectivity with face4 is observed only when face $3 b$ is considered separately. Moreover, there are few nodes that show significant connectivity only when the entire $3 \mathrm{~b}$ is considered to together (for details see Fig. 6).

Some of the salient features of the functional networks are described below.

\subsubsection{Connectivity with contralateral $3 b$}

Different body part representations showed differences in the extent of connectivity with homotopic representations in the contralateral 3b. All ROI's in humans showed significant bilateral connectivity (Fig. 6b). Average Fisher's ztransformed correlation coefficient $(\mathrm{CCz})$ values in humans showed that the 
highest inter-hemispherical correlation was for face $3 b$, followed by med $3 b$ and hand3b (Fig. 7; face3b: $p=6.7 \times 10^{-10}$; med3b: $p=2.3 \times 10^{-9}$; hand3b: $p=4.03 \times$ $10^{-7}$; one sample t-test). The ROI's in monkeys although did not have statistically

\section{a. Macaque monkeys}

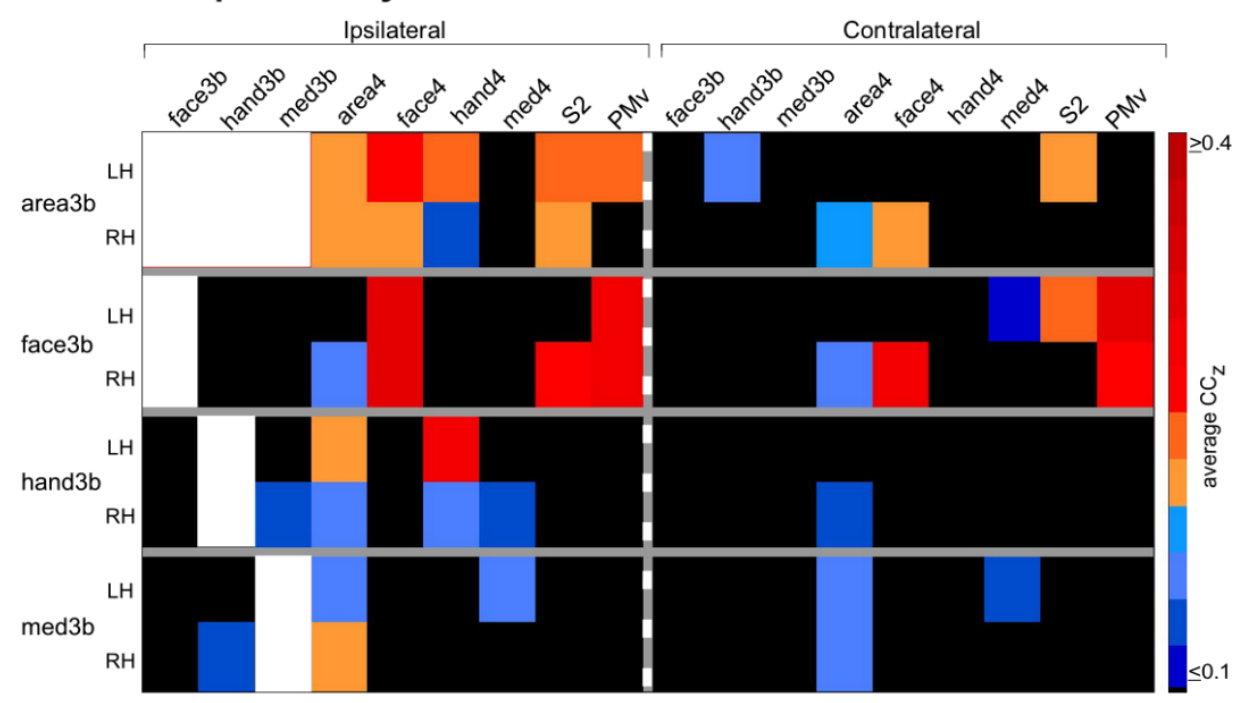

b. Humans

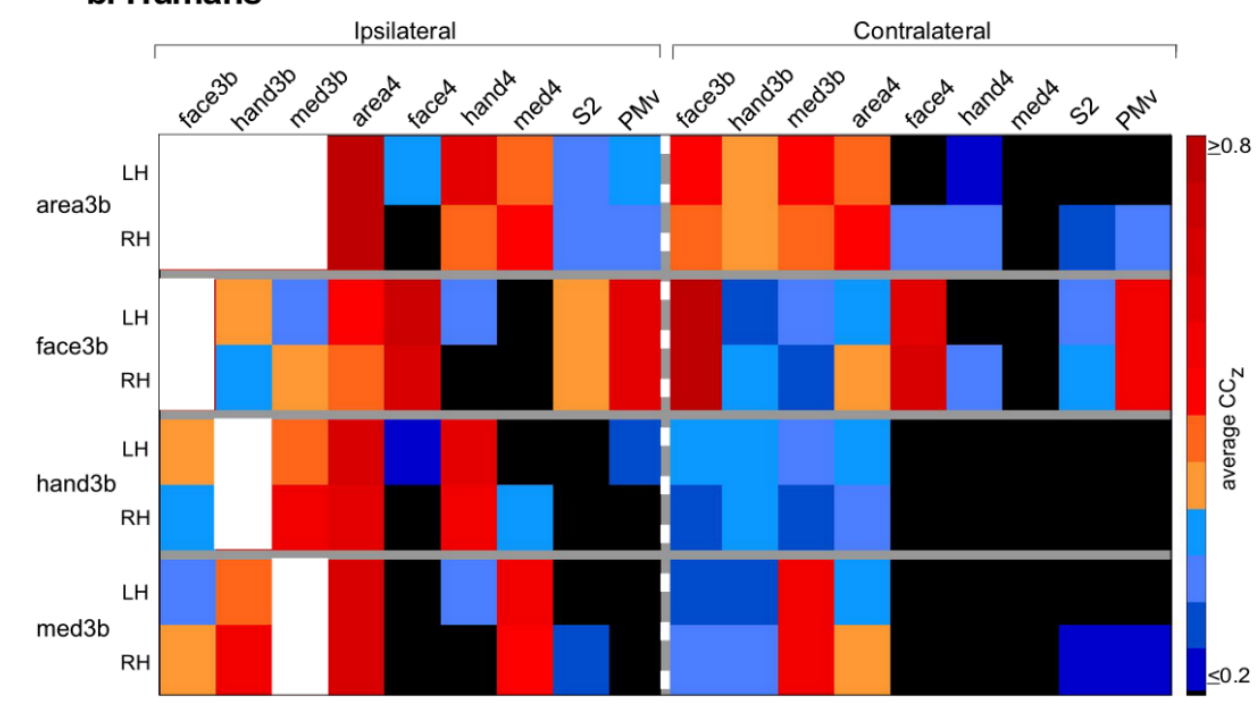

Fig. 6. Correlation matrices showing ROI-ROI resting state functional connectivity between different ROI's in ipsilateral (left of the dashed white line) and contralateral hemispheres (right of the dashed white line) of (a) monkeys and (b) humans. The seed regions are shown on the $y$-axis, and the target regions on top. Each colour coded value denotes averaged Fisher-z transformed correlations $(\mathrm{CCz})$ acquired from different subjects. For colour codes, see the bars on the right. Statistically nonsignificant correlations are shown in black and autocorrelations are in white ( $p \leq 0.05$ for monkeys; $p \leq 0.00001$ for humans; one sample t-test). 3b, area 3b; 4, area 4; 'face', 'hand', 'med' prefixes denote the face, hand and the medial part of area $3 \mathrm{~b}$ or 4 . $\mathrm{LH}$, left hemisphere; $\mathrm{RH}$, right hemisphere. 
significant homotopic bilateral connectivity, but followed similar trend in the strength of connectivity (Fig. 7; face3b: $p=6.9 \times 10^{-2}$; $\operatorname{med} 3 \mathrm{~b}: p=1.08 \times 10^{-1}$; hand3b: $p=2.43 \times 10^{-1}$; one sample t-test).

The interhemispheric connectivity of all the three ROI's - face $3 b$, hand $3 b$ and med $3 b$ with non-homotopic ROI's in contralateral area $3 b$ was alsosignificant only in humans (Fig. 6 and 10; one-sample t-test, see Table 2 for $p$-values).

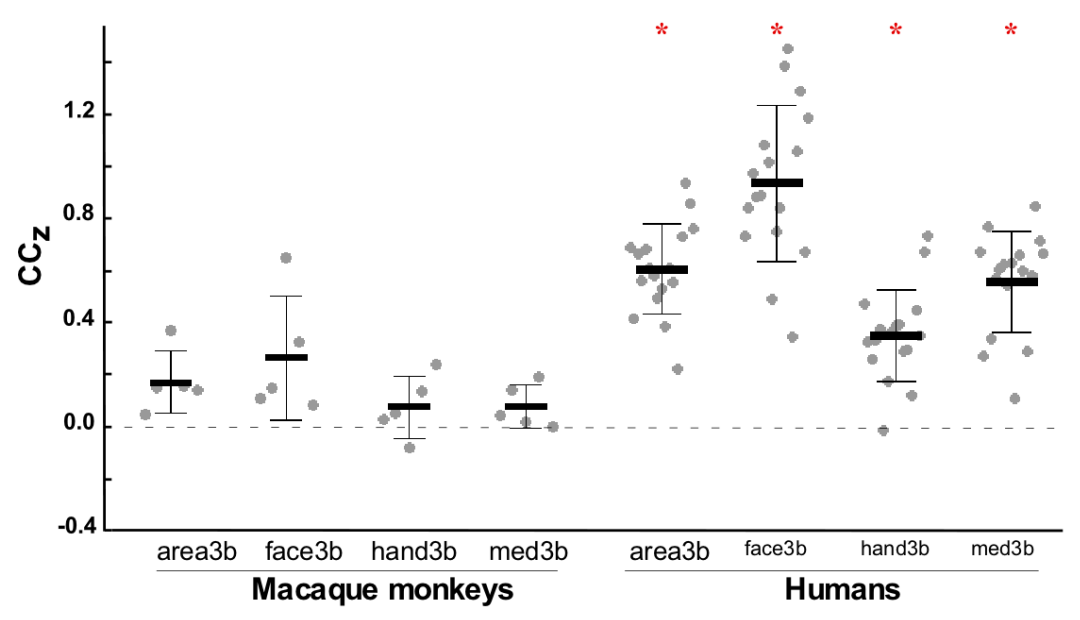

Fig. 7. Inter-hemispheric homotopic functional connectivity of somatosensory area $3 b$, and ROI's for different body part representations in macaque monkeys and humans. Each dot on the plot denotes Fisher-z transformed correlation value $\left(\mathrm{CC}_{\mathrm{Z}}\right)$ for a single subject in monkeys and humans. Thick horizontal lines shows the mean and the thin lines, \pm SD. Asterisk* denotes significant statistical difference $(p<0.00001$ in humans, one sample t-test).

\subsubsection{Functional connectivity with $\mathrm{S} 2$ and PMv}

Our ROI's in S2 and PMv encompassed the entire areas; no attempt was made to place ROI's in representations of specific body parts. Therefore, the connectivity of individual body part representations that we refer to below is with the entire area S2 or PMv.

Face $3 b$ of humans in both hemispheres had significant connectivity with S2 bilaterally (see Fig. 8a for ipsilateral connectivity, LH, $p=3.08 \times 10^{-6}$; RH, $p=$ $1.0 \times 10^{-8}$; for $p$ values for contralateral S2 see Table 2). However, in monkeys face $3 b$ of the right hemisphere had significant connectivity only with ipsilateral 
S2 (Fig. 8a; $p=2.56 \times 10^{-2}$ ), whereas the face $3 b$ of the left hemisphere had significant connectivity with only the contralateral S2 (Fig. $6 ; p=2.12 \times 10^{-2}$ ). Thus, $\mathrm{S} 2$ of only the right hemisphere was connected to face $3 \mathrm{~b}$.

The hand $3 b$ of either monkeys or humans did not have significant connectivity with S2 (Fig. 8a; for monkeys LH, $p=3.15 \times 10^{-1}$; RH, $p=4.18 \times 10^{-1}$; for humans LH, $p=1.22 \times 10^{-4}$; RH, $p=1.05 \times 10^{-4}$; one sample t-test). Similar to hand $3 b$, the med $3 b$ of monkeys showed no significant connectivity with S2 (Fig. $8 \mathrm{a}$; LH, $p=3.0 \times 10^{-1}$; RH, $p=3.05 \times 10^{-1}$ ). However, in humans med3b of the right hemisphere had bilateral connectivity with $\mathrm{S} 2$, whereas med3b of the left hemispheres had no significant connectivity with S2 (Fig. 8a; LH, $p=3.89 \times 10^{-5}$; RH, ipsilateral, $p=4.48 \times 10^{-6}$, contralateral (see Fig 6b), $p=8.91 \times 10^{-6}$; one sample t-test).

With PMv, face $3 \mathrm{~b}$ of both monkeys and humans had significant connectivity (Fig. 8b; for monkeys LH, $p=2.1 \times 10^{-2}$; RH, $p=3.74 \times 10^{-2}$; for humans $\mathrm{LH}, p=$ $4.71 \times 10^{-9}$; RH, $p=3=8.76 \times 10^{-9}$; one sample t-test; for $p$ values for contralateral PMv see Table 1 and 2). In monkeys the hand3b or med3b did not show any significant connectivity with PMv. In humans the hand $3 \mathrm{~b}$ of the left hemisphere had significant connectivity with ipsilateral PMv (Fig. 8b, top; $p=8.4 \times 10^{-6}$ ), and med $3 b$ of only the right hemisphere had significant connectivity but only with contralateral PMv (Fig. 6b; $p=8.94 \times 10^{-6}$ ).

The data suggested that in both the species the observed connectivity for the entire area $3 b$ with $S 2$ and PMv was primarily a reflection of the face $3 b$ correlations with these areas.

\subsubsection{Connectivity with homotopic representations in area 4}

We compared the correlations of different body part representations in area $3 b$ 
with the homotopic body part representation in motor area 4. All three ROIs face $3 b$, hand $3 b$ and med $3 b$ showed strong connectivity with the corresponding representation in the ipsilateral primary motor cortex in both macaque monkeys and humans, except for the right med3b of monkeys (Fig. 6). Interestingly, the mean correlation strength of face $3 b$ with face 4 was significantly higher than the correlation of face $3 b$ with hand $3 b$ or med3b (see Fig. 9, left, for LH, macaque, $F_{(2,81)}=25.84, p<0.0001$; humans, $F_{(2,51)}=35.40, p<0.0001$; two-way ANOVA; $p<0.01$, post hoc Tukey test; see Fig. 9, right, for RH, macaque, $F_{(2,81)}$ $=13.79, p<0.0001$; humans, $p=F_{(2,51)}=30.56, p<0.0001 ;$ two-way ANOVA; $p$ $<0.01$, post hoc Tukey test). Thus, connectivity of face representation in area $3 \mathrm{~b}$ was stronger with face representation in area 4 than for other ROI's in area $3 b$.

In the left hemisphere of both the species, the connectivity of hand3b with hand 4 was significantly higher than with face 3 b or med3b (For LH, macaque, $F_{(2,81)}=$ 22.86, $p<0.0001$; humans, $F_{(2,51)}=12.05, p<0.0001$; two-way ANOVA; $p<$ 0.01 , post hoc Tukey test). In both species, the connectivity of hand $3 b$ with hand 4 in the right hemisphere was higher, but not statistically significantly when compared to connectivity with face $3 b$ or with med3b (For $\mathrm{RH}$, macaque, $F_{(2,81)}=$ 2.37, $p=0.10$; humans, $F_{(2,51)}=5.93, p<0.004$; two-way ANOVA; n.s, post hoc Tukey test).

Connectivity of med3b with med4 in humans was significantly higher than with face $3 b$ or hand $3 b$, but only in the left hemisphere $\left(F_{(2,51)}=12.56, p<0.0001\right.$; two-way ANOVA; $p<0.01$, post hoc Tukey test). The mean connectivity of med3b with med4 in the right hemisphere of both species was higher than with face $3 b$ or with hand $3 b$ but the difference was not statistically significant (for RH, macaque, $F_{(2,81)}=0.845, p=0.4334$; humans, $F_{(2,51)}=7.55, p=0.0013$; twoway ANOVA; n.s, post hoc Tukey test). 


\subsection{Comparison of the functional connectivity in monkeys and humans}

The functional networks of entire area $3 \mathrm{~b}$ and different body part representations had many similarities in both macaque monkeys and humans (Fig. 4 and 10). The

a. Correlation with $\mathbf{S 2}$
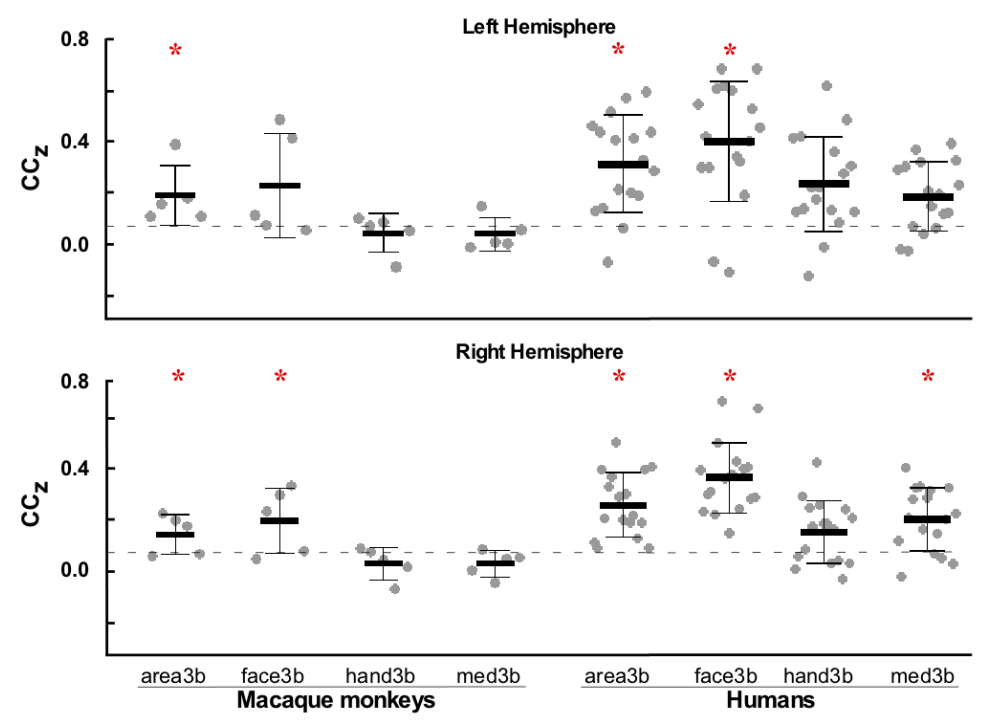

b. Correlation with PMv
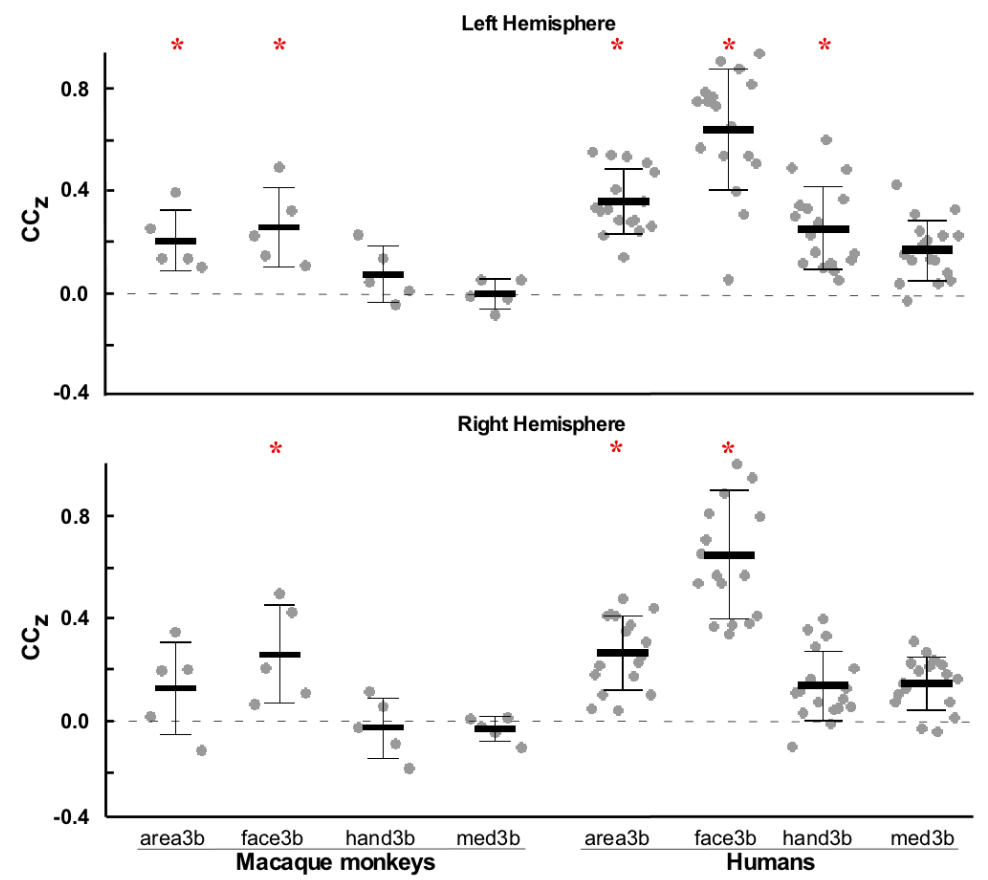

Fig. 8. Correlation coefficients (CCz) of somatosensory ROIs with ipsilateral (a) S2 and (b) $\mathrm{PMv}$ in macaque monkeys and humans. Each dot on the plot denotes Fisher-z transformed correlation value $(\mathrm{CCz})$ for a single subject in monkeys and humans. Horizontal lines show the mean (thick lines) and \pm S.D (thin lines) of correlation coefficients. Asterisk* denotes statistical significant difference ( $p<0.05$ in monkeys; $p<0.00001$ in humans, one sample ttest). 
network if determined for entire area $3 \mathrm{~b}$ largely represents nodes of individual networks of face $3 b$, hand $3 b$ and med $3 b$. In both monkeys and humans, face $3 b$ had functional connectivity with regions in the primary motor cortex as well as other areas. Only the face $3 \mathrm{~b}$ was functionally connected to S2 and PMv, bilaterally for humans and in the right hemisphere for monkeys (Fig. 8). The hand $3 \mathrm{~b}$ and med $3 \mathrm{~b}$ of both the hemispheres in both species had network connectivity restricted to area $3 \mathrm{~b}$ and area 4 (Fig. 6).

However, there were few notable differences in the functionally connected regions and their connectivity strengths between the two species. Face $3 b$ connections in macaque monkeys showed significant bilateral correlations with parietal area $7 \mathrm{~b}$ and putamen, which were not observed in humans (Fig. 5). The face $3 b$ network in monkeys did not show any connections with the regions on the medial wall at the border of area 4 and the supplementary motor area, which was seen in humans (Fig. 5).
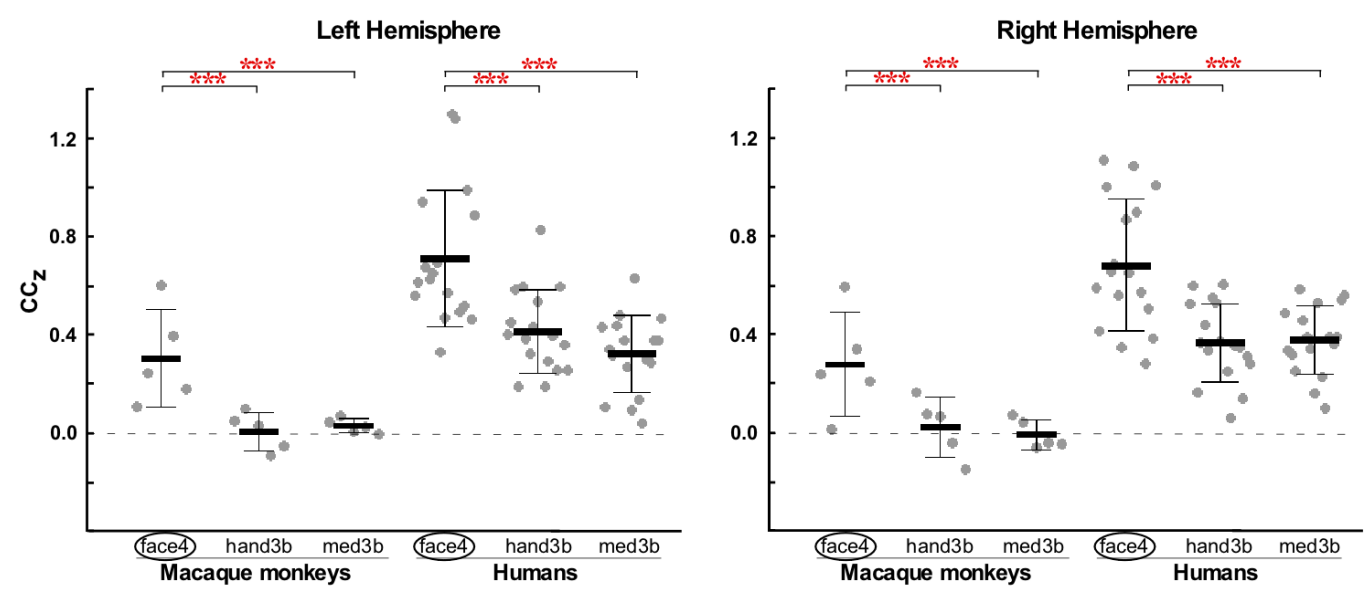

Fig. 9. Functional connectivity of somatosensory face representation, face3b with rostrally adjacent homotopic motor representation in area 4 (face4; enclosed in oval) and its comparison with connectivity with other representations (hand3b and med3b) in the same hemisphere of macaque monkeys and humans. Data is shown for both the hemispheres. Each dot on the plot denotes Fisher-z transformed correlation value $(C C z)$ for a single subject in monkeys and humans. Horizontal lines show the mean (thick line) and \pm S.D (thin line) of the connectivity strength. Asterisks ${ }^{* * *}$ denote statistically significant difference, $p<$ 0.0001 , two-way ANOVA (main effect of ROI's); $p<0.01$, post hoc Tukey test. 
Significant connectivity of the body part representations in area $3 b$ to homotopic representations in contralateral area 3b was observed only in humans (Fig. 6 and 10). Finally, the significant connectivity between different body part representations within area $3 \mathrm{~b}$ i.e. face, hand and medial regions was also observed only in humans (Fig. 6 and 10).

\section{DISCUSSION}

We performed seed-based correlation analysis to determine resting-state connectivity of different body part representations in area $3 \mathrm{~b}$ of macaque monkeys and humans. Previously both somatosensory and motor areas have been lumped together for connectivity analysis. Our results show that there is a characteristic somatosensory network for each body part representation, which is largely similar in macaque monkeys and humans. Some of the main observations from our ROI-ROI and ROI-voxel analyses are discussed below. The results are summarized in Figure 10.

\subsection{Understanding brain connectivity using resting-state correlations}

Resting-state functional connectivity networks, which are determined from the time-series correlation of different brain regions in a task free condition have been described for different mammalian species including humans, monkeys, cats, ferrets, rats and mice (Biswal et al., 1995; Lu et al., 2012; Popa et al., 2009; Stafford et al., 2014; Vincent et al., 2007; Zhou et al., 2016). Resting-state networks have been used to delineate functional brain networks such as somatomotor, visual and auditory networks, the dorsal and ventral attention systems, the fronto-opercular salience region, and what is known as default mode network (Biswal et al., 1995; Cordes et al., 2000; De Luca et al., 2005; Fox et al., 2006; Fox et al., 2005; Greicius et al., 2003; Lowe et al., 1998; Raichle et al., 2001; Seeley et al., 2007). The resting-state networks also predict brain areas that 
would be active during stimulus driven activation and during various cognitive tasks (De Luca et al., 2005; Hampson et al., 2006; Seeley et al., 2007; Tavor et al., 2016; Vincent et al., 2006).

We determined resting-state network connectivity for different topographic representations in the somatosensory cortex of macaque monkeys and humans. Previous reports in macaque monkeys described the resting-state network considering all the somatomotor areas as a single ROI (Hutchison et al., 2011), or at the most after dividing the somatomotor areas into dorsal and ventral subdivisions (Mantini et al., 2013). We show here that each body part representation in area $3 \mathrm{~b}$ has its own distinct network, which is different from that for other body parts. Network of the entire area $3 \mathrm{~b}$ largely reflects sum of all the individual networks. The somatosensory network for complete area $3 \mathrm{~b}$ described here is generally similar to as those for the 'somatomotor networks' described in previous studies (Biswal et al., 1995; Kuehn et al., 2017; Mantini et al., 2013; Vincent et al., 2007). However, some of the nodes such as area 5, 7, putamen, and insula are revealed only when area $3 \mathrm{~b}$ was considered separately as seed ROI, suggesting importance of area specific ROI's for connectivity analysis.

Previously, neuroimaging studies have divided area $3 \mathrm{~b}$ into two independent networks - a ventral network that comprises of the tongue and possibly lower face representation, and a medial network comprised of the hand and other body part representations (Power et al., 2011; Yeo et al., 2011). These were defined by network parcellation. We show here that the face representation, even after excluding the intraoral structures is an independent network separate from the 'hand network'. It is likely that the face and oral structures would form separate networks, given they have different connectivity (Cerkevich et al., 2014; Iyengar et al., 2007) and are revealed as separate myelin rich modules in histological preparations of the monkey cortex (Jain et al., 2001). 
We predict that if we determine functional connectivity of cortical representations at higher granularity, more details of connectivity will be revealed. For example, each digit might reveal a specific connectivity due to digit specific, and divergent and convergent connections (Ashaber et al., 2014; Negyessy et al., 2013; Wang et al., 2013). At an even higher resolution different parts of the digits, e.g. proximal and distal likely reveal different connectivity (Liao et al., 2013). Furthermore, each of the body part representation in our med3b will likely have different connectivity pattern (Krubitzer and Kaas, 1990).

\subsection{Functional connectivity reflects anatomical connections}

The resting-state functional networks reflect anatomical connections as determined using neuroanatomical tracers or lesion studies. Relationship between functional connectivity and direct anatomical connectivity has been emphasized (van den Heuvel et al., 2009; Wang et al., 2013). While anatomical connections in monkeys have been described in detail, information on the anatomical connectivity in humans is sparse and generally indirectly inferred. Our results show that in monkeys area $3 \mathrm{~b}$ has connectivity with areas $1,2,5,7, \mathrm{~S} 2 / \mathrm{PV}, \mathrm{PMv}$ and insula, the areas that are known to have anatomical connections with area $3 b$ (Burton et al., 1995; Darian-Smith et al., 1993; Jones and Powell, 1969a; Pons and Kaas, 1986). Differences in the ipsilateral and contralateral resting-state connectivity observed for different body part representations - face $3 b$, hand $3 b$, and med $3 \mathrm{~b}$ reflects differences in the underlying anatomical connectivity of these representations (Jones and Powell, 1969b; Killackey et al., 1983). For example, we found that face $3 b$ network has the largest number of bilateral nodes which include face4, S2/PV, PMv and insular cortex, and the contralateral face $3 \mathrm{~b}$. This reflects anatomical connectivity of face representation in area $3 b$. Face representation in area $3 b$ is known to receive direct projections from area 3a, 1, 2, S2/PV, PMv and Insula (Cerkevich et al., 2014; Disbrow et al., 2003). 
Connectivity of area3b is also seen with areas 3a, 1 and 2 (see Fig. 4 and 5) but is not separately analyzed here because of difficulties in placing ROI's that are clearly distinct from area $3 b$.

In macaque monkeys, the face representation also showed significant correlation with the posterior parietal area $7 \mathrm{~b}$ and putamen in the ROI to voxel analysis. Neuroanatomical studies have shown connectivity of the face representation in area $3 b$ with the rostro-lateral part of area $7 b$ (Burton et al., 1995; Lewis and Van Essen, 2000), and large parts of area $7 \mathrm{~b}$ has somatosensory responses to mouth and face (Hyvarinen, 1981). In monkeys topographic projections from the somatosensory cortex to putamen have been seen using anatomical tracers (Jones et al., 1977; Kunzle, 1977) and in lesion studies (Kemp and Powell, 1970). The voxels in the lateral putamen correlating with face $3 \mathrm{~b}$ are in a similar location as the somatosensory projections to putamen (Kunzle, 1977).

As compared to face $3 b$, the network of other ROIs i.e., hand $3 b$ and med $3 b$ had fewer nodes, which were largely confined to the primary motor cortex (see Results, and Fig. 10). In monkeys, hand $3 b$ did not functionally connect to the hand representation in contralateral area $3 \mathrm{~b}$. This reflects the lack of homotopic callosal connections of the hand representation in area 3b (Killackey et al., 1983). In monkeys, bilateral correlation of hand $3 b$ with area 5 was observed. In area 5 there are converging inputs in the forelimb representation with neurons having large receptive fields on the arm and the hand (Padberg et al., 2005; Seelke et al., 2012).

Differences in the functional connectivity of the face and hand representations in area $3 \mathrm{~b}$ with PMv also reflect differences in the anatomical connectivity. Although the strongest projections from area $3 b$ to PMv are from the face representation, PMv also has minor inputs from the hand representation (Dancause et al., 2006). In humans we observed hand3b to PMv connectivity in the left hemisphere. 
In both species, the bilateral connectivity of area $3 \mathrm{~b}$ representations to homotopic representations in the contralateral hemisphere is in agreement with the known callosal connectivity of body part representation in area $3 \mathrm{~b}$, where face and trunk representations have more callosal connections as compared to the hand representation (Killackey et al., 1983; Krubitzer and Kaas, 1990; Pandya and Vignolo, 1969). Moreover, the midline body parts such as the face and the trunk have occasional bilateral receptive fields in area 3b (Conti et al., 1986; Dreyer et al., 1975; Eickhoff et al., 2008; Fabri et al., 2005). Although most of these seems to be contributed by callosal connections (Fabri et al., 2006) peripheral contribution cannot be ruled out (Iwamura et al., 2001). These peripheral bilateral inputs can also possibly contribute to interhemispheric connectivity. Thus, the functional connectivity of different body part representations reflects anatomical connectivity.

However, we did not observe any significant resting-state correlations of hand representation in area $3 \mathrm{~b}$ with $\mathrm{S} 2$ in spite of their direct anatomical connectivity (Eickhoff et al., 2007; Krubitzer et al., 1995). Among the five monkeys scanned, the hand3b-S2 correlation was found only in two animals using seed-to-voxel analysis at lenient thresholds and does not emerge as significant correlation in the ROI-ROI analysis. In humans also, the hand $3 b$ had correlations to S2 at lenient thresholds observed only in the seed-to-voxel analysis.

\subsection{Functional connectivity is not always dependent upon direct structural connectivity}

It has been suggested that functional connectivity is not necessarily constrained by absence of direct structural connectivity (Raichle, 2015). The functional connectivity networks are not only driven by monosynaptic connections but also polysynaptic connections (Honey et al., 2009; Vincent et al., 2007). Thus restingstate coherence observed between areas that lack monosynaptic connections 
likely reflects emergent cortical network properties (Adachi et al., 2012; Vincent et al., 2007). Our results also reveal this aspect of structure-function relationship of functional networks. For example, we found strong functional association between area $3 \mathrm{~b}$ and area 4 representations, which lack direct anatomical
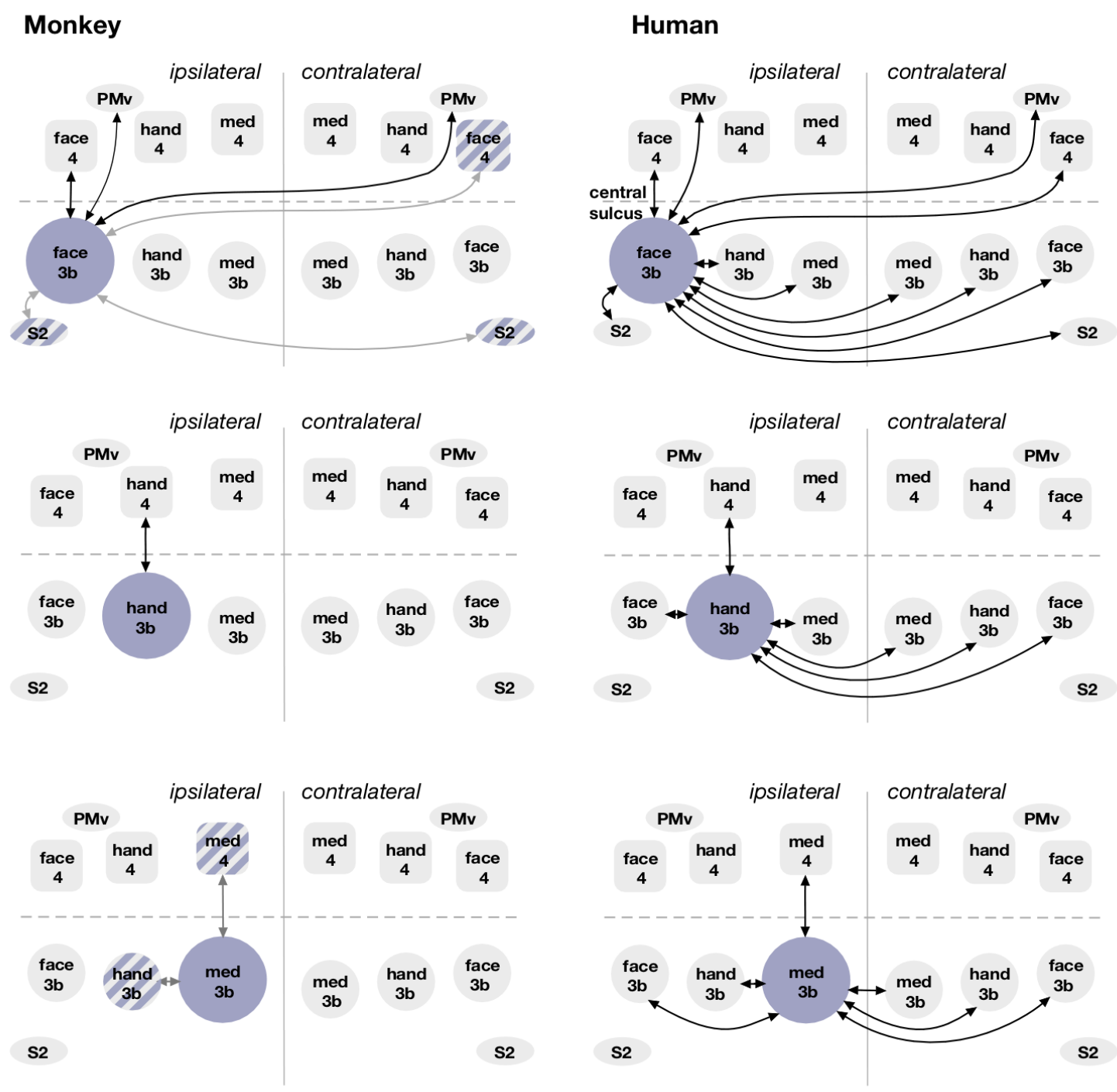

Fig. 10. A schematic showing resting-state functional connectivity of different ROl's - face3b, hand3b, and med3b (large circles) in the ipsilateral and contralateral hemispheres in macaque monkeys (left panels) and humans (right panels). Significant connections common to both hemispheres are depicted. Spheres represent topographic representations in area $3 b$, rounded squares in area 4; and ovals represent areas other than the primary cortices. Blue shaded regions show areas with statistically significant ROI-ROI correlations as shown by double headed arrows. The regions filled with grey did not have any significant correlations. In addition, significant connections in one hemisphere of monkeys but both hemispheres of humans are shown for comparison by grey lines and hashed fills. One sample t-test, $p<0.05$ (monkeys), $p<0.00001$ (humans). Arrows in the schematic do not imply any directionality. 
connections. These functional correlations might reflect the indirect anatomical links through somatosensory area 2 and 5 which receive projections from area $3 \mathrm{~b}$ and send projections to the motor cortex (Darian-Smith et al., 1993; Jones et al., 1978; Vogt and Pandya, 1978).

The functional connectivity between representations across the central sulcus points to information flow between homologous representations in area $3 \mathrm{~b}$ and area 4 required for coordinated activity in these brain areas required for sensorimotor tasks. There is also a phylogenetic closeness of the neuronal mass populating the similar body representations that mediate coordinated neural activity in the sensory and motor regions (Flechsig, 1920 as cited in Kuehn et al., 2017). A functional association between motor and somatosensory areas has been observed as spatiotemporal coherence in local field potential (LFP) signals (ArceMcShane et al., 2016; Murthy and Fetz, 1992).

There are other examples illustrating that resting-state functional connectivity also reflects indirect or higher order anatomical connectivity. For example, connectivity of area $3 \mathrm{~b}$ with insular cortex is likely a reflection of indirect anatomical connectivity via the secondary somatosensory cortex (Burton et al., 1995; Friedman et al., 1986). Functionally, innocuous somatosensory stimulation of face is known to elicit neuronal responses in the granular regions of insula (Schneider et al., 1993).

\subsection{Resting networks of area $3 \mathrm{~b}$ are similar in humans and monkeys}

Although separated by 25 million years of evolution (Kaas, 2004, 2012), macaque monkeys and humans share similarities in the structural connectivity and functional networks of different brain areas (Goulas et al., 2014; Mantini et al., 2013; Sallet et al., 2013). The somatomotor networks have high topological correspondence in humans and monkeys (Mantini et al., 2013). The current study is the first to determine and directly compare resting-state networks of different 
topographic representations in both humans and macaque monkeys. We used somatotopy specific seed ROIs that were also validated using localizer scans in both macaques and humans, assuring accuracy of the observed networks.

Our results show that connectivity of different body part representations in area 3 biz. face $3 b$, hand $3 b$ and med $3 b$ is similar in monkeys and humans (see Fig. 5, 6). These similarities include (1) face 3 b having widespread functional connectivity with networks that include ipsilateral face4, bilateral PMv, and the insular cortex, and (2) functional connectivity of the face and hand representations in area $3 \mathrm{~b}$ with homotopic representations in area 4 . These similarities likely reflect many behavioral similarities between the monkeys and humans. Both these species are highly social with a variety of complex facial expressions that are important for maintaining the social structure (Burrows, 2008). The widespread functional associations seen especially for the face representation in both monkeys and humans might underlie the important role of facial structures in verbal and nonverbal communication which enables the rich socio-cultural lives of primate communities. Although the difference in the ability to use the hand vary considerably, monkeys are able to make a large variety for grasps just like humans (Macfarlane and Graziano, 2009). Both these species have opposable thumbs enabling fine grasping ability (Marzke, 1997).

Previous neuroanatomical data from monkeys has shown that connectivity between different areas is strongest between homotopic body part representations (Ashaber et al., 2014; Negyessy et al., 2013; Wang et al., 2013). Our results also show stronger functional connectivity between homotopic body part representations, thus reinforcing the importance of somatotopic representations in information processing.

There were also certain differences in the functional connectivity networks of two primate species (see Fig. 5, 10), probably reflecting species specific behavioral 
differences. Many functional correlations were found only in humans. For example, humans showed correlation of face3b with voxels at M1/SMA border on the medial wall while the monkey brains did not. Bilateral connections between homologous area $3 \mathrm{~b}$ representations were observed only in humans; as well as the significant connectivity between face, hand and the medial regions of area $3 \mathrm{~b}$ was present only in humans. The comparatively higher connectivity of face representation in humans could be a reflection of the importance of facial gestures for emotions and social cues. The bilateral connectivity of the somatosensory representations in humans reflects a wider repertoire of behavior in humans that uses bilateral coordination in bipedal humans including their complex object manipulation abilities that requires fine tactile inputs.

\subsection{Limitations of the study and methodological considerations}

The difference in functional connectivity between humans and monkeys could reflect evolutionary differences between the two species. However, there are technical considerations that should also be taken into account while interpreting the results.

The inter-species differences in functional connectivity could be due to differences in the brain organization i.e. the number and sizes of the cortical areas, their specialization, and anatomical connectivity. For example, correlations of area $3 \mathrm{~b}$ representations with putamen, area 7 and area 5 were found only in macaque monkeys. These, differences in functional connectivity might also be due to the differences in brain sizes of the two species which can give rise to alterations in neuronal wiring and information processing networks (Kaas, 2000).

The mean correlation strengths of connections in monkeys were always lower than in humans (e.g. Figs 7, $8 \&$ 9). Larger size of human brain along with its dense white matter fiber connections can give rise to stronger correlation values. 
However, the correlation strength difference could also be due to differences in the sizes of the monkey and human brain scanned in the same magnet. Although we used a knee coil for monkeys to improve the filling factor, the signal to noise ratio was better for the human brain.

Difference in the brain states can also result in the difference between the human and monkey data. We scanned monkeys in anesthetized state for ease of handling, while human participants were awake. Because of the anesthetized state of monkeys, there could be a reduction in the BOLD signal strength and consequently reduction in the calculated correlation strengths (Bettinardi et al., 2015; Grandjean et al., 2014; Vincent et al., 2007). Isoflurane, used in the current study, is known to depress the brain activity and cause general nonselective suppressive effects on local functional connectivity of fine-scale cortical circuits in a dose dependent manner (Hutchison et al., 2014; Wu et al., 2016). Thus, the functional connectivity determined during the awake state might be reflected as widespread interareal connectivity only in humans, but could become more restricted in anaesthetized monkeys.

Different anesthetic agents affect the connectivity patterns differently (Paasonen et al., 2018). Ketamine, an NMDA antagonist, has been shown to reduce the intrinsic brain connectivity in primary somatosensory and auditory cortices (Niesters et al., 2012). We chose isoflurane because comparative studies using different anesthetic agents show that isoflurane is an ideal candidate for restingstate connectivity studies in animal models (Grandjean et al., 2014; Jonckers et al., 2014; Lv et al., 2016). Moreover, the isoflurane levels in the current study ( $0.8 \%$ maximum) were lower than the most resting-state studies which use up to 1.25\%-1.5\% isoflurane (Hutchison et al., 2014; Vincent et al., 2007; Wu et al., 2016). Although the amplitude of functional correlations might reduce under anesthesia, the conservation of functional networks across different brain states such as arousal and sleep have been described (Fukunaga et al., 2006; Hutchison 
et al., 2013). This suggests that despite the differences in the brain state while scanning, the differences observed between the two species could be actual species-specific differences.

Although there are reports that the functional connectivity is not static but dynamically variable across different time scales (Chang and Glover, 2010; Hutchison et al., 2013), we used averaged connectivity measures from each ROI for the analysis. Certainly, different analysis methods considering non-stationary transient state changes in connectivity can give more complete information regarding the baseline spontaneous activity in the brain. However, the topographic variation of resting functional networks across different body part representations was visible even while using zero-lag, time averaged correlation measures. Despite these limitations we found remarkable similarities between humans and monkeys.

\subsection{CONCLUSIONS}

Our results show that the network architecture described as 'somatomotor network' is a composite structure and is comprised of multiple independent and different networks of different topographic representations. Care needs to be taken while assigning the network characteristics uniformly to the participating nodes. A similar recent study has described the non-uniformity of the task negative default mode network (DMN) in humans and has found that subnetworks exist within the DMN (Braga and Buckner, 2017). Previous reports on the intrinsic functional connectivity of human brain discuss the intra network heterogeneity within somatomotor network and suggest the importance of topographic areas in delineating network boundaries (Kuehn et al., 2017; Long et al., 2014; Power et al., 2011; Yeo et al., 2011). The current study extends the understanding of the normal structure of spontaneous connectivity and describes how it varies across different body part representations in somatosensory area $3 \mathrm{~b}$ 
in macaque monkeys and humans. In all the acquired fMRI sessions in both the species, the somatosensory ROIs consistently described distinct functional subnetworks, which largely reflected the underlying anatomical connectivity patterns. The results suggest that rather than considering the entire 'somatomotor' area as a whole, connectivity network analysis should take body part representations into consideration. This knowledge of the somatotopy dependent connectivity is crucial in understanding changes in the information processing within these networks in disease and injury conditions such as spinal cord injury that cause large-scale somatotopic reorganization.

\section{COMPETING FINANCIAL INTERESTS}

The authors declare no competing financial interests.

\section{ACKNOWLEDGEMENTS}

Authors are grateful for funding by Department of Biotechnology, Government of India to N.J. (No.BT/PR7180/MED/30/907/2012) and core funds from National Brain Research Centre. We also thank Mr. Atanu Datta, Mr. Arun E V R and Mr. Raghav Shankar for assistance with acquisition of monkey fMRI data, and Mr. Jitender Ahlawat for technical assistance. We thank Dr. V Rema for helpful comments on the manuscript. 
Table 1. $p$-values for ROI-ROI correlations for monkeys. LH, left hemisphere; RH, right hemisphere. Only significant pair-wise correlations i.e. where $p$-value $<0.05$ (one sample t-test) are shown. Grey boxes are auto-correlations.

\begin{tabular}{|c|c|c|c|c|c|c|c|c|c|}
\hline \multirow{2}{*}{\multicolumn{2}{|c|}{ Target }} & \multicolumn{2}{|c|}{ area3b } & \multicolumn{2}{|c|}{ face3b } & \multicolumn{2}{|c|}{ hand3b } & \multicolumn{2}{|c|}{ med3b } \\
\hline & & $\mathrm{RH}$ & $\mathrm{LH}$ & $\mathrm{RH}$ & $\mathrm{LH}$ & $\mathrm{RH}$ & $\mathrm{LH}$ & $\mathrm{RH}$ & $\mathrm{LH}$ \\
\hline \multirow{2}{*}{ face $3 b$} & $\mathrm{RH}$ & $5.0 \times 10^{-04}$ & - & & - & - & - & - & - \\
\hline & $\mathrm{LH}$ & - & $1.3 \times 10^{-03}$ & & & - & - & - & - \\
\hline \multirow{2}{*}{ hand3b } & $\mathrm{RH}$ & $1.85 \times 10^{-08}$ & $1.43 \times 10^{-02}$ & & & & - & $1.13 \times 10^{-02}$ & - \\
\hline & $\mathrm{LH}$ & - & $1.27 \times 10^{-06}$ & & & & & - & - \\
\hline \multirow{2}{*}{ med3b } & $\mathrm{RH}$ & $2.0 \times 10^{-04}$ & - & & & & & & - \\
\hline & $\mathrm{LH}$ & - & $4.0 \times 10^{-04}$ & & & & & & \\
\hline \multirow{2}{*}{ face 4} & $\mathrm{RH}$ & $4.56 \times 10^{-02}$ & - & $2.66 \times 10^{-02}$ & - & - & - & - & - \\
\hline & $\mathrm{LH}$ & $9.0 \times 10^{-03}$ & $2.98 \times 10^{-02}$ & $1.46 \times 10^{-02}$ & $4.26 \times 10^{-02}$ & - & - & - & - \\
\hline \multirow{2}{*}{ hand4 } & $\mathrm{RH}$ & $2.61 \times 10^{-02}$ & - & - & - & $5.3 \times 10^{-03}$ & - & - & - \\
\hline & $\mathrm{LH}$ & - & $8.5 \times 10^{-03}$ & - & - & - & $1.90 \times 10^{-03}$ & - & - \\
\hline \multirow{2}{*}{ med4 } & $\mathrm{RH}$ & - & - & - & $1.21 \times 10^{-02}$ & $1.44 \times 10^{-02}$ & - & - & $2.31 \times 10^{-02}$ \\
\hline & $\mathrm{LH}$ & - & - & - & - & - & - & - & $2.14 \times 10^{-02}$ \\
\hline \multirow{2}{*}{ S2 } & $\mathrm{RH}$ & $1.39 \times 10^{-02}$ & $2.21 \times 10^{-02}$ & $2.56 \times 10^{-02}$ & $2.12 \times 10^{-02}$ & - & - & - & - \\
\hline & LH & - & $2.56 \times 10^{-02}$ & - & - & - & - & - & - \\
\hline \multirow{2}{*}{ PMv } & $\mathrm{RH}$ & - & - & $3.74 \times 10^{-02}$ & $4.63 \times 10^{-02}$ & - & - & - & - \\
\hline & $\mathrm{LH}$ & - & $2.05 \times 10^{-02}$ & $2.61 \times 10^{-02}$ & $2.1 \times 10^{-02}$ & - & - & - & - \\
\hline
\end{tabular}


Table 2. $p$-values for ROI-ROI correlations for humans. LH, left hemisphere; RH, right hemisphere. Only significant pair-wise correlations i.e. where $p$-value $<0.00001$ (one sample ttest) are shown. Grey boxes are auto-correlations.

\begin{tabular}{|c|c|c|c|c|c|c|c|c|c|}
\hline \multirow{2}{*}{\multicolumn{2}{|c|}{ Target }} & \multicolumn{2}{|c|}{ area3b } & \multicolumn{2}{|c|}{ face $3 b$} & \multicolumn{2}{|c|}{ hand3b } & \multicolumn{2}{|c|}{ med3b } \\
\hline & & $\mathrm{RH}$ & $\mathrm{LH}$ & $\mathrm{RH}$ & $\mathrm{LH}$ & $\mathrm{RH}$ & $\mathrm{LH}$ & $\mathrm{RH}$ & $\mathrm{LH}$ \\
\hline \multirow[b]{2}{*}{ face $3 b$} & $\mathrm{RH}$ & $3.70 \times 10^{-12}$ & $3.13 \times 10^{-09}$ & & $6.66 \times 10^{-10}$ & $5.80 \times 10^{-08}$ & $3.80 \times 10^{-06}$ & $5.86 \times 10^{-09}$ & $6.21 \times 10^{-06}$ \\
\hline & $\mathrm{LH}$ & $3.19 \times 10^{-08}$ & $5.85 \times 10^{-12}$ & & & $4.82 \times 10^{-06}$ & $1.91 \times 10^{-08}$ & $6.70 \times 10^{-06}$ & $1.71 \times 10^{-07}$ \\
\hline \multirow[b]{2}{*}{ hand3b } & $\mathrm{RH}$ & $6.94 \times 10^{-14}$ & $1.91 \times 10^{-08}$ & & & & $4.03 \times 10^{-07}$ & $8.03 \times 10^{-10}$ & $3.51 \times 10^{-07}$ \\
\hline & $\mathrm{LH}$ & $8.38 \times 10^{-09}$ & $1.92 \times 10^{-12}$ & & & & & $5.15 \times 10^{-06}$ & $8.35 \times 10^{-09}$ \\
\hline \multirow[b]{2}{*}{ med3b } & $\mathrm{RH}$ & $5.40 \times 10^{-14}$ & $1.66 \times 10^{-09}$ & & & & & & $2.34 \times 10^{-09}$ \\
\hline & $\mathrm{LH}$ & $1.00 \times 10^{-08}$ & $1.68 \times 10^{-15}$ & & & & & & \\
\hline \multirow[b]{2}{*}{ face4 } & $\mathrm{RH}$ & - & - & $1.18 \times 10^{-08}$ & $2.53 \times 10^{-06}$ & - & - & - & - \\
\hline & $\mathrm{LH}$ & $5.90 \times 10^{-08}$ & $1.92 \times 10^{-08}$ & $9.59 \times 10^{-09}$ & $1.29 \times 10^{-08}$ & - & $9.64 \times 10^{-06}$ & - & - \\
\hline \multirow[b]{2}{*}{ hand4 } & $\mathrm{RH}$ & $2.84 \times 10^{-07}$ & $1.40 \times 10^{-06}$ & - & - & $1.07 \times 10^{-07}$ & - & - & - \\
\hline & $\mathrm{LH}$ & $8.16 \times 10^{-06}$ & $1.68 \times 10^{-08}$ & $2.44 \times 10^{-06}$ & $2.72 \times 10^{-07}$ & - & $2.77 \times 10^{-08}$ & - & $1.35 \times 10^{-07}$ \\
\hline \multirow[b]{2}{*}{ med4 } & $\mathrm{RH}$ & $3.39 \times 10^{-07}$ & - & - & - & $9.68 \times 10^{-07}$ & - & $2.52 \times 10^{-06}$ & - \\
\hline & $\mathrm{LH}$ & - & $1.79 \times 10^{-07}$ & - & - & - & - & - & $3.56 \times 10^{-07}$ \\
\hline \multirow[b]{2}{*}{$\mathbf{S 2}$} & $\mathrm{RH}$ & $2.54 \times 10^{-07}$ & - & $1.00 \times 10^{-08}$ & $4.87 \times 10^{-06}$ & - & - & $4.48 \times 10^{-06}$ & - \\
\hline & $\mathrm{LH}$ & $5.04 \times 10^{-07}$ & $4.36 \times 10^{-06}$ & $5.86 \times 10^{-07}$ & $3.08 \times 10^{-06}$ & - & - & $8.91 \times 10^{-06}$ & - \\
\hline \multirow[b]{2}{*}{ PMv } & $\mathrm{RH}$ & $9.49 \times 10^{-07}$ & - & $8.76 \times 10^{-09}$ & $1.73 \times 10^{-06}$ & - & - & - & - \\
\hline & $\mathrm{LH}$ & $1.21 \times 10^{-07}$ & $2.52 \times 10^{-09}$ & $1.46 \times 10^{-08}$ & $4.71 \times 10^{-09}$ & - & $8.40 \times 10^{-06}$ & $8.94 \times 10^{-06}$ & - \\
\hline
\end{tabular}




\section{REFERENCES}

Adachi, Y., Osada, T., Sporns, O., Watanabe, T., Matsui, T., Miyamoto, K., Miyashita, Y., 2012. Functional connectivity between anatomically unconnected areas is shaped by collective network-level effects in the macaque cortex. Cereb Cortex 22, 1586-1592.

Amunts, K., Zilles, K., 2015. Architectonic Mapping of the Human Brain beyond Brodmann. Neuron 88, 1086-1107.

Arce-McShane, F.I., Ross, C.F., Takahashi, K., Sessle, B.J., Hatsopoulos, N.G., 2016. Primary motor and sensory cortical areas communicate via spatiotemporally coordinated networks at multiple frequencies. Proc Natl Acad Sci U S A 113, 5083-5088.

Ashaber, M., Palfi, E., Friedman, R.M., Palmer, C., Jakli, B., Chen, L.M., Kantor, O., Roe, A.W., Negyessy, L., 2014. Connectivity of somatosensory cortical area 1 forms an anatomical substrate for the emergence of multifinger receptive fields and complex feature selectivity in the squirrel monkey (Saimiri sciureus). J Comp Neurol 522, 1769-1785.

Bakker, R., Tiesinga, P., Kotter, R., 2015. The Scalable Brain Atlas: Instant WebBased Access to Public Brain Atlases and Related Content. Neuroinformatics 13, 353-366.

Barbas, H., Pandya, D.N., 1987. Architecture and frontal cortical connections of the premotor cortex (area 6) in the rhesus monkey. J Comp Neurol 256, 211-228.

Beckmann, C.F., DeLuca, M., Devlin, J.T., Smith, S.M., 2005. Investigations into resting-state connectivity using independent component analysis. Philos Trans $\mathrm{R}$ Soc Lond B Biol Sci 360, 1001-1013.

Behzadi, Y., Restom, K., Liau, J., Liu, T.T., 2007. A component based noise correction method (CompCor) for BOLD and perfusion based fMRI. Neuroimage 37, 90-101.

Bettinardi, R.G., Tort-Colet, N., Ruiz-Mejias, M., Sanchez-Vives, M.V., Deco, G., 2015. Gradual emergence of spontaneous correlated brain activity during fading of general anesthesia in rats: Evidences from fMRI and local field potentials. Neuroimage 114, 185-198.

Biswal, B., Zerrin Yetkin, F., Haughton, V.M., Hyde, J.S., 1995. Functional connectivity in the motor cortex of resting human brain using echo- planar mri. Magn Reson Med 34, 537-541. 
Blankenburg, F., Ruben, J., Meyer, R., Schwiemann, J., Villringer, A., 2003. Evidence for a rostral-to-caudal somatotopic organization in human primary somatosensory cortex with mirror-reversal in areas $3 \mathrm{~b}$ and 1 . Cereb Cortex 13 , 987-993.

Blatow, M., Nennig, E., Durst, A., Sartor, K., Stippich, C., 2007. fMRI reflects functional connectivity of human somatosensory cortex. Neuroimage 37, 927936.

Braga, R.M., Buckner, R.L., 2017. Parallel Interdigitated Distributed Networks within the Individual Estimated by Intrinsic Functional Connectivity. Neuron 95, 457-471 e455.

Brodmann, K., 1909. Vergleichende Lokalisationslehre der Grosshirnrinde in ihren Prinzipien dargestellt auf Grund des Zellenbaues. Barth.

Burrows, A.M., 2008. The facial expression musculature in primates and its evolutionary significance. Bioessays 30, 212-225.

Burton, H., Fabri, M., Alloway, K., 1995. Cortical areas within the lateral sulcus connected to cutaneous representations in areas $3 \mathrm{~b}$ and 1 : a revised interpretation of the second somatosensory area in macaque monkeys. J Comp Neurol 355, 539562.

Cerkevich, C.M., Qi, H.X., Kaas, J.H., 2014. Corticocortical projections to representations of the teeth, tongue, and face in somatosensory area $3 \mathrm{~b}$ of macaques. J Comp Neurol 522, 546-572.

Chand, P., Jain, N., 2015. Intracortical and Thalamocortical Connections of the Hand and Face Representations in Somatosensory Area 3b of Macaque Monkeys and Effects of Chronic Spinal Cord Injuries. J Neurosci 35, 13475- 13486.

Chang, C., Glover, G.H., 2010. Time-frequency dynamics of resting-state brain connectivity measured with fMRI. Neuroimage 50, 81-98.

Choe, A.S., Jones, C.K., Joel, S.E., Muschelli, J., Belegu, V., Caffo, B.S., Lindquist, M.A., van Zijl, P.C., Pekar, J.J., 2015. Reproducibility and Temporal Structure in Weekly Resting-State fMRI over a Period of 3.5 Years. PLoS One 10, e0140134.

Conti, F., Fabri, M., Manzoni, T., 1986. Bilateral receptive fields and callosal connectivity of the body midline representation in the first somatosensory area of primates. Somatosens Res 3, 273-289. 
Cordes, D., Haughton, V.M., Arfanakis, K., Wendt, G.J., Turski, P.A., Moritz, C.H., Quigley, M.A., Meyerand, M.E., 2000. Mapping functionally related regions of brain with functional connectivity MR imaging. AJNR Am J Neuroradiol 21, 1636-1644.

Damoiseaux, J.S., Greicius, M.D., 2009. Greater than the sum of its parts: a review of studies combining structural connectivity and resting-state functional connectivity. Brain Struct Funct 213, 525-533.

Dancause, N., Barbay, S., Frost, S.B., Plautz, E.J., Stowe, A.M., Friel, K.M., Nudo, R.J., 2006. Ipsilateral connections of the ventral premotor cortex in a new world primate. J Comp Neurol 495, 374-390.

Darian-Smith, C., Darian-Smith, I., Burman, K., Ratcliffe, N., 1993. Ipsilateral cortical projections to areas $3 \mathrm{a}, 3 \mathrm{~b}$, and 4 in the macaque monkey. J Comp Neurol $335,200-213$.

De Luca, M., Smith, S., De Stefano, N., Federico, A., Matthews, P.M., 2005. Blood oxygenation level dependent contrast resting state networks are relevant to functional activity in the neocortical sensorimotor system. Exp Brain Res 167, 587-594.

Disbrow, E., Litinas, E., Recanzone, G.H., Padberg, J., Krubitzer, L., 2003. Cortical connections of the second somatosensory area and the parietal ventral area in macaque monkeys. J Comp Neurol 462, 382-399.

Disbrow, E., Roberts, T., Krubitzer, L., 2000. Somatotopic organization of cortical fields in the lateral sulcus of Homo sapiens: evidence for SII and PV. J Comp Neurol 418, 1-21.

Dreyer, D.A., Loe, P.R., Metz, C.B., Whitsel, B.L., 1975. Representation of head and face in postcentral gyrus of the macaque. J Neurophysiol 38, 714-733

Dutta, A., Kambi, N., Raghunathan, P., Khushu, S., Jain, N., 2014. Large- scale reorganization of the somatosensory cortex of adult macaque monkeys revealed by fMRI. Brain Struct Funct 219, 1305-1320.

Eickhoff, S.B., Grefkes, C., Fink, G.R., Zilles, K., 2008. Functional lateralization of face, hand, and trunk representation in anatomically defined human somatosensory areas. Cereb Cortex 18, 2820-2830.

Eickhoff, S.B., Grefkes, C., Zilles, K., Fink, G.R., 2007. The somatotopic organization of cytoarchitectonic areas on the human parietal operculum. Cereb Cortex 17, 1800-1811. 
Eickhoff, S.B., Stephan, K.E., Mohlberg, H., Grefkes, C., Fink, G.R., Amunts, K., Zilles, K., 2005. A new SPM toolbox for combining probabilistic cytoarchitectonic maps and functional imaging data. Neuroimage 25, 1325-1335.

Fabri, M., Polonara, G., Mascioli, G., Paggi, A., Salvolini, U., Manzoni, T., 2006. Contribution of the corpus callosum to bilateral representation of the trunk midline in the human brain: an fMRI study of callosotomized patients. Eur $\mathbf{J}$ Neurosci 23, 3139-3148.

Fabri, M., Polonara, G., Salvolini, U., Manzoni, T., 2005. Bilateral cortical representation of the trunk midline in human first somatic sensory area. Hum Brain Mapp 25, 287-296.

Fang, P.C., Jain, N., Kaas, J.H., 2002. Few intrinsic connections cross the handface border of area $3 b$ of New World monkeys. J Comp Neurol 454, 310- 319.

Ferretti, A., Del Gratta, C., Babiloni, C., Caulo, M., Arienzo, D., Tartaro, A., Rossini, P.M., Romani, G.L., 2004. Functional topography of the secondary somatosensory cortex for nonpainful and painful stimulation of median and tibial nerve: an fMRI study. Neuroimage 23, 1217-1225.

Fonov, V.S., Evans, A.C., McKinstry, R.C., Almli, C.R., Collins, D.L., 2009. Unbiased nonlinear average age-appropriate brain templates from birth to adulthood. Neuroimage 47, S102.

Fox, M.D., Corbetta, M., Snyder, A.Z., Vincent, J.L., Raichle, M.E., 2006. Spontaneous neuronal activity distinguishes human dorsal and ventral attention systems. Proc Natl Acad Sci U S A 103, 10046-10051.

Fox, M.D., Snyder, A.Z., Vincent, J.L., Corbetta, M., Van Essen, D.C., Raichle, M.E., 2005. The human brain is intrinsically organized into dynamic, anticorrelated functional networks. Proc Natl Acad Sci U S A 102, 9673-9678.

Friedman, D.P., Murray, E.A., O'Neill, J.B., Mishkin, M., 1986. Cortical connections of the somatosensory fields of the lateral sulcus of macaques: evidence for a corticolimbic pathway for touch. J Comp Neurol 252, 323-347.

Fukunaga, M., Horovitz, S.G., van Gelderen, P., de Zwart, J.A., Jansma, J.M., Ikonomidou, V.N., Chu, R., Deckers, R.H., Leopold, D.A., Duyn, J.H., 2006. Large-amplitude, spatially correlated fluctuations in BOLD fMRI signals during extended rest and early sleep stages. Magn Reson Imaging 24, 979-992.

Geyer, S., 2004. The microstructural border between the motor and the cognitive domain in the human cerebral cortex. Adv Anat Embryol Cell Biol 174, I-VIII, 189. 
Geyer, S., Matelli, M., Luppino, G., Zilles, K., 2000a. Functional neuroanatomy of the primate isocortical motor system. Anat Embryol (Berl) 202, 443-474.

Geyer, S., Schleicher, A., Zilles, K., 1999. Areas 3a, 3b, and 1 of human primary somatosensory cortex. Neuroimage 10, 63-83.

Geyer, S., Schormann, T., Mohlberg, H., Zilles, K., 2000b. Areas 3a, 3b, and 1 of human primary somatosensory cortex. Part 2. Spatial normalization to standard anatomical space. Neuroimage 11, 684-696.

Glasser, M.F., Coalson, T.S., Robinson, E.C., Hacker, C.D., Harwell, J., Yacoub, E., Ugurbil, K., Andersson, J., Beckmann, C.F., Jenkinson, M., Smith, S.M., Van Essen, D.C., 2016. A multi-modal parcellation of human cerebral cortex. Nature $536,171-178$.

Gordon, E.M., Laumann, T.O., Adeyemo, B., Huckins, J.F., Kelley, W.M., Petersen, S.E., 2016. Generation and Evaluation of a Cortical Area Parcellation from Resting-State Correlations. Cereb Cortex 26, 288-303.

Goulas, A., Bastiani, M., Bezgin, G., Uylings, H.B., Roebroeck, A., Stiers, P., 2014. Comparative analysis of the macroscale structural connectivity in the macaque and human brain. PLoS Comput Biol 10, e1003529.

Grandjean, J., Schroeter, A., Batata, I., Rudin, M., 2014. Optimization of anesthesia protocol for resting-state fMRI in mice based on differential effects of anesthetics on functional connectivity patterns. Neuroimage $102 \mathrm{Pt} 2$, 838- 847.

Greicius, M.D., Krasnow, B., Reiss, A.L., Menon, V., 2003. Functional connectivity in the resting brain: a network analysis of the default mode hypothesis. Proc Natl Acad Sci U S A 100, 253-258.

Hampson, M., Driesen, N.R., Skudlarski, P., Gore, J.C., Constable, R.T., 2006. Brain connectivity related to working memory performance. J Neurosci 26 , 13338-13343.

Honey, C.J., Sporns, O., Cammoun, L., Gigandet, X., Thiran, J.P., Meuli, R., Hagmann, P., 2009. Predicting human resting-state functional connectivity from structural connectivity. Proc Natl Acad Sci U S A 106, 2035-2040.

Hutchison, R.M., Hutchison, M., Manning, K.Y., Menon, R.S., Everling, S., 2014. Isoflurane induces dose-dependent alterations in the cortical connectivity profiles and dynamic properties of the brain's functional architecture. Hum Brain Mapp 35, 5754-5775. 
Hutchison, R.M., Leung, L.S., Mirsattari, S.M., Gati, J.S., Menon, R.S., Everling, S., 2011. Resting-state networks in the macaque at 7 T. Neuroimage 56, 15461555.

Hutchison, R.M., Womelsdorf, T., Gati, J.S., Everling, S., Menon, R.S., 2013. Resting-state networks show dynamic functional connectivity in awake humans and anesthetized macaques. Hum Brain Mapp 34, 2154-2177.

Hyvarinen, J., 1981. Regional distribution of functions in parietal association area 7 of the monkey. Brain Res 206, 287-303.

Iyengar, S., Qi, H.X., Jain, N., Kaas, J.H., 2007. Cortical and thalamic connections of the representations of the teeth and tongue in somatosensory cortex of new world monkeys. J Comp Neurol 501, 95-120.

Iwamura, Y., Taoka, M., Iriki, A., 2001. Bilateral activity and callosal connections in the somatosensory cortex. Neuroscientist 7, 419-429.

Jain, N., Catania, K.C., Kaas, J.H., 1998. A histologically visible representation of the fingers and palm in primate area $3 \mathrm{~b}$ and its immutability following longterm deafferentations. Cereb Cortex 8, 227-236.

Jain, N., Preuss, T.M., Kaas, J.H., 1994. Subdivisions of the visual system labeled with the Cat-301 antibody in tree shrews. Vis Neurosci 11, 731-741.

Jain, N., Qi, H.X., Catania, K.C., Kaas, J.H., 2001. Anatomic correlates of the face and oral cavity representations in the somatosensory cortical area $3 \mathrm{~b}$ of monkeys. J Comp Neurol 429, 455-468.

Jain, N., Qi, H.X., Collins, C.E., Kaas, J.H., 2008. Large-scale reorganization in the somatosensory cortex and thalamus after sensory loss in macaque monkeys. $\mathrm{J}$ Neurosci 28, 11042-11060.

Jenkinson, M., Bannister, P., Brady, M., Smith, S., 2002. Improved optimization for the robust and accurate linear registration and motion correction of brain images. Neuroimage 17, 825-841.

Jonckers, E., Delgado y Palacios, R., Shah, D., Guglielmetti, C., Verhoye, M., Van der Linden, A., 2014. Different anesthesia regimes modulate the functional connectivity outcome in mice. Magn Reson Med 72, 1103-1112.

Jones, E.G., Coulter, J.D., Burton, H., Porter, R., 1977. Cells of origin and terminal distribution of corticostriatal fibers arising in the sensory-motor cortex of monkeys. J Comp Neurol 173, 53-80. 
Jones, E.G., Coulter, J.D., Hendry, S.H., 1978. Intracortical connectivity of architectonic fields in the somatic sensory, motor and parietal cortex of monkeys. J Comp Neurol 181, 291-347.

Jones, E.G., Powell, T.P., 1969a. Connexions of the somatic sensory cortex of the rhesus monkey. I. Ipsilateral cortical connexions. Brain 92, 477-502.

Jones, E.G., Powell, T.P., 1969b. Connexions of the somatic sensory cortex of the rhesus monkey. II. Contralateral cortical connexions. Brain 92, 717-730.

Kaas, J.H., 2000. Why is Brain Size so Important:Design Problems and Solutions as Neocortex Gets Bigger or Smaller. Brain and Mind 1, 7-23.

Kaas, J.H., 2004. Evolution of somatosensory and motor cortex in primates. Anat Rec A Discov Mol Cell Evol Biol 281, 1148-1156.

Kaas, J.H., 2012. The evolution of neocortex in primates. Prog Brain Res 195, 91102.

Kambi, N., Halder, P., Rajan, R., Arora, V., Chand, P., Arora, M., Jain, N., 2014. Large-scale reorganization of the somatosensory cortex following spinal cord injuries is due to brainstem plasticity. Nat Commun 5, 3602.

Kambi, N., Tandon, S., Mohammed, H., Lazar, L., Jain, N., 2011. Reorganization of the primary motor cortex of adult macaque monkeys after sensory loss resulting from partial spinal cord injuries. J Neurosci 31, 3696-3707.

Kemp, J.M., Powell, T.P., 1970. The cortico-striate projection in the monkey. Brain 93, 525-546.

Killackey, H.P., Gould, H.J., 3rd, Cusick, C.G., Pons, T.P., Kaas, J.H., 1983. The relation of corpus callosum connections to architectonic fields and body surface maps in sensorimotor cortex of new and old world monkeys. J Comp Neurol 219, 384-419.

Krubitzer, L., Clarey, J., Tweedale, R., Elston, G., Calford, M., 1995. A redefinition of somatosensory areas in the lateral sulcus of macaque monkeys. $\mathrm{J}$ Neurosci 15, 3821-3839.

Krubitzer, L.A., Kaas, J.H., 1990. The organization and connections of somatosensory cortex in marmosets. J Neurosci 10, 952-974.

Kuehn, E., Dinse, J., Jakobsen, E., Long, X., Schafer, A., Bazin, P.L., Villringer, A., Sereno, M.I., Margulies, D.S., 2017. Body Topography Parcellates Human Sensory and Motor Cortex. Cereb Cortex 27, 3790-3805. 
Kunzle, H., 1977. Projections from the primary somatosensory cortex to basal ganglia and thalamus in the monkey. Exp Brain Res 30, 481-492.

Ladak, A., Tubbs, R.S., Spinner, R.J., 2014. Mapping sensory nerve communications between peripheral nerve territories. Clin Anat 27, 681-690

Laumann, T.O., Gordon, E.M., Adeyemo, B., Snyder, A.Z., Joo, S.J., Chen, M.Y., Gilmore, A.W., McDermott, K.B., Nelson, S.M., Dosenbach, N.U., Schlaggar, B.L., Mumford, J.A., Poldrack, R.A., Petersen, S.E., 2015.Functional System and Areal Organization of a Highly Sampled Individual Human Brain. Neuron 87, 657-670.

Lewis, J.W., Van Essen, D.C., 2000. Corticocortical connections of visual, sensorimotor, and multimodal processing areas in the parietal lobe of the macaque monkey. Journal of Comparative Neurology 428, 112-137.

Liao, C.C., Gharbawie, O.A., Qi, H., Kaas, J.H., 2013. Cortical connections to single digit representations in area $3 \mathrm{~b}$ of somatosensory cortex in squirrel monkeys and prosimian galagos. J Comp Neurol 521, 3768-3790.

Long, X., Goltz, D., Margulies, D.S., Nierhaus, T., Villringer, A., 2014. Functional connectivity-based parcellation of the human sensorimotor cortex. Eur J Neurosci 39, 1332-1342.

Lowe, M.J., Mock, B.J., Sorenson, J.A., 1998. Functional connectivity in single and multislice echoplanar imaging using resting-state fluctuations. Neuroimage 7 , 119-132.

Lu, H., Zou, Q., Gu, H., Raichle, M.E., Stein, E.A., Yang, Y., 2012. Rat brains also have a default mode network. Proc Natl Acad Sci U S A 109, 3979- 3984.

Lv, P., Xiao, Y., Liu, B., Wang, Y., Zhang, X., Sun, H., Li, F., Yao, L., Zhang, W., Liu, L., Gao, X., Wu, M., Tang, Y., Chen, Q., Gong, Q., Lui, S., 2016. Dosedependent effects of isoflurane on regional activity and neural network function: A resting-state fMRI study of 14 rhesus monkeys: An observational study.

Neurosci Lett 611, 116-122.

Macfarlane, N.B., Graziano, M.S., 2009. Diversity of grip in Macaca mulatta. Exp Brain Res 197, 255-268.

Maldjian, J.A., Gottschalk, A., Patel, R.S., Detre, J.A., Alsop, D.C., 1999. The sensory somatotopic map of the human hand demonstrated at 4 Tesla.

Neuroimage 10, 55-62. 
Manger, P.R., Woods, T.M., Jones, E.G., 1996. Representation of face and intraoral structures in area $3 \mathrm{~b}$ of macaque monkey somatosensory cortex. J Comp Neurol 371, 513-521.

Manger, P.R., Woods, T.M., Munoz, A., Jones, E.G., 1997. Hand/face border as a limiting boundary in the body representation in monkey somatosensory cortex. $\mathbf{J}$ Neurosci 17, 6338-6351.

Mantini, D., Corbetta, M., Romani, G.L., Orban, G.A., Vanduffel, W., 2013. Evolutionarily novel functional networks in the human brain? J Neurosci 33, 3259-3275.

Marzke, M.W., 1997. Precision grips, hand morphology, and tools. Am J Phys Anthropol 102, 91-110

Miyamoto, J.J., Honda, M., Saito, D.N., Okada, T., Ono, T., Ohyama, K., Sadato, N., 2006. The representation of the human oral area in the somatosensory cortex: a functional MRI study. Cereb Cortex 16, 669-675.

Murthy, V.N., Fetz, E.E., 1992. Coherent 25- to 35-Hz oscillations in the sensorimotor cortex of awake behaving monkeys. Proc Natl Acad Sci U S A 89, $5670-5674$.

Nakamura, A., Yamada, T., Goto, A., Kato, T., Ito, K., Abe, Y., Kachi, T., Kakigi, R., 1998. Somatosensory homunculus as drawn by MEG. Neuroimage 7, 377-386.

Negyessy, L., Palfi, E., Ashaber, M., Palmer, C., Jakli, B., Friedman, R.M., Chen, L.M., Roe, A.W., 2013. Intrinsic horizontal connections process global tactile features in the primary somatosensory cortex: neuroanatomical evidence. J Comp Neurol 521, 2798-2817.

Nelson, R.J., Sur, M., Felleman, D.J., Kaas, J.H., 1980. Representations of the body surface in postcentral parietal cortex of Macaca fascicularis. J Comp Neurol 192, 611-643.

Niesters, M., Khalili-Mahani, N., Martini, C., Aarts, L., van Gerven, J., van Buchem, M.A., Dahan, A., Rombouts, S., 2012. Effect of subanesthetic ketamine on intrinsic functional brain connectivity: a placebo-controlled functional magnetic resonance imaging study in healthy male volunteers. Anesthesiology $117,868-877$. 
Paasonen, J., Stenroos, P., Salo, R.A., Kiviniemi, V., Grohn, O., 2018. Functional connectivity under six anesthesia protocols and the awake condition in rat brain. Neuroimage 172, 9-20.

Padberg, J., Disbrow, E., Krubitzer, L., 2005. The organization and connections of anterior and posterior parietal cortex in titi monkeys: do New World monkeys have an area 2? Cereb Cortex 15, 1938-1963.

Pandya, D.N., Vignolo, L.A., 1969. Interhemispheric projections of the parietal lobe in the rhesus monkey. Brain Res 15, 49-65.

Paxinos, G., Huang, X.-F., Toga, A.W., 2000. The rhesus monkey brain in stereotaxic coordinates.

Pons, T.P., Kaas, J.H., 1986. Corticocortical connections of area 2 of somatosensory cortex in macaque monkeys: a correlative anatomical and electrophysiological study. J Comp Neurol 248, 313-335.

Pons, T.P., Wall, J.T., Garraghty, P.E., Cusick, C.G., Kaas, J.H., 1987. Consistent features of the representation of the hand in area $3 \mathrm{~b}$ of macaque monkeys.

Somatosens Res 4, 309-331.

Popa, D., Popescu, A.T., Pare, D., 2009. Contrasting activity profile of two distributed cortical networks as a function of attentional demands. J Neurosci 29, 1191-1201.

Power, J.D., Cohen, A.L., Nelson, S.M., Wig, G.S., Barnes, K.A., Church, J.A., Vogel, A.C., Laumann, T.O., Miezin, F.M., Schlaggar, B.L., Petersen, S.E., 2011. Functional network organization of the human brain. Neuron 72, 665-678.

Preuss, T.M., Stepniewska, I., Jain, N., Kaas, J.H., 1997. Multiple divisions of macaque precentral motor cortex identified with neurofilament antibody SMI-32. Brain Res 767, 148-153.

Pruessmann, K.P., Weiger, M., Scheidegger, M.B., Boesiger, P., 1999. SENSE: sensitivity encoding for fast MRI. Magn Reson Med 42, 952-962.

Raichle, M.E., 2015. The restless brain: how intrinsic activity organizes brain function. Philos Trans R Soc Lond B Biol Sci 370.

Raichle, M.E., MacLeod, A.M., Snyder, A.Z., Powers, W.J., Gusnard, D.A., Shulman, G.L., 2001. A default mode of brain function. Proc Natl Acad Sci U S A 98, 676-682. 
Rohlfing, T., Kroenke, C.D., Sullivan, E.V., Dubach, M.F., Bowden, D.M., Grant, K.A., Pfefferbaum, A., 2012. The INIA19 Template and NeuroMaps Atlas for Primate Brain Image Parcellation and Spatial Normalization. Front Neuroinform 6, 27.

Rorden, C., Brett, M., 2000. Stereotaxic display of brain lesions. Behavioural neurology 12, 191-200.

Saleem, K.S., Logothetis, N.K., 2006. Atlas of the rhesus monkey brain in stereotaxic coordinates: a combined mri and histology. Academic Press.

Sallet, J., Mars, R.B., Noonan, M.P., Neubert, F.X., Jbabdi, S., O'Reilly, J.X., Filippini, N., Thomas, A.G., Rushworth, M.F., 2013. The organization of dorsal frontal cortex in humans and macaques. J Neurosci 33, 12255-12274.

Schieber, M.H., 2001. Constraints on somatotopic organization in the primary motor cortex. J Neurophysiol 86, 2125-2143.

Schneider, R.J., Friedman, D.P., Mishkin, M., 1993. A modality-specific somatosensory area within the insula of the rhesus monkey. Brain Res 621, 116120.

Seeley, W.W., Menon, V., Schatzberg, A.F., Keller, J., Glover, G.H., Kenna, H., Reiss, A.L., Greicius, M.D., 2007. Dissociable intrinsic connectivity networks for salience processing and executive control. J Neurosci 27, 2349- 2356.

Seelke, A.M., Padberg, J.J., Disbrow, E., Purnell, S.M., Recanzone, G., Krubitzer, L., 2012. Topographic Maps within Brodmann's Area 5 of macaque monkeys. Cereb Cortex 22, 1834-1850.

Shehzad, Z., Kelly, A.M., Reiss, P.T., Gee, D.G., Gotimer, K., Uddin,L.Q., Lee, S.H., Margulies, D.S., Roy, A.K., Biswal, B.B., Petkova, E., Castellanos, F.X., Milham, M.P., 2009. The resting brain: unconstrained yet reliable. Cereb Cortex 19, 2209-2229.

Sohn, W.S., Yoo, K., Jeong, Y., 2012. Independent component analysis of localized resting-state functional magnetic resonance imaging reveals specific motor subnetworks. Brain Connect 2, 218-224.

Stafford, J.M., Jarrett, B.R., Miranda-Dominguez, O., Mills, B.D., Cain, N., Mihalas, S., Lahvis, G.P., Lattal, K.M., Mitchell, S.H., David, S.V., Fryer, J.D., Nigg, J.T., Fair, D.A., 2014. Large-scale topology and the default mode network in the mouse connectome. Proc Natl Acad Sci U S A 111, 18745- 18750. 
Stepniewska, I., Preuss, T.M., Kaas, J.H., 1993. Architectonics, somatotopic organization, and ipsilateral cortical connections of the primary motor area (M1) of owl monkeys. J Comp Neurol 330, 238-271.

Tandon, S., Kambi, N., Lazar, L., Mohammed, H., Jain, N., 2009. Large-scale expansion of the face representation in somatosensory areas of the lateral sulcus after spinal cord injuries in monkeys. J Neurosci 29, 12009-12019.

Tavor, I., Parker Jones, O., Mars, R.B., Smith, S.M., Behrens, T.E., Jbabdi, S., 2016. Task-free MRI predicts individual differences in brain activity during task performance. Science 352, 216-220.

Van den Heuvel, M.P., Mandl, R.C., Kahn, R.S., Hulshoff Pol, H.E., 2009. Functionally linked resting-state networks reflect the underlying structural connectivity architecture of the human brain. Hum Brain Mapp 30, 3127-3141.

Van Essen, D.C., Drury, H.A., Dickson, J., Harwell, J., Hanlon, D., Anderson, C.H., 2001. An integrated software suite for surface-based analyses of cerebral cortex. J Am Med Inform Assoc 8, 443-459.

Van Essen, D.C., Glasser, M.F., Dierker, D.L., Harwell, J., 2012. Cortical parcellations of the macaque monkey analyzed on surface-based atlases. Cereb Cortex 22, 2227-2240.

Vincent, J.L., Patel, G.H., Fox, M.D., Snyder, A.Z., Baker, J.T., Van Essen, D.C., Zempel, J.M., Snyder, L.H., Corbetta, M., Raichle, M.E., 2007. Intrinsic functional architecture in the anaesthetized monkey brain. Nature 447, 83-86.

Vincent, J.L., Snyder, A.Z., Fox, M.D., Shannon, B.J., Andrews, J.R., Raichle, M.E., Buckner, R.L., 2006. Coherent spontaneous activity identifies a hippocampal-parietal memory network. J Neurophysiol 96, 3517-3531.

Vogt, B.A., Pandya, D.N., 1978. Cortico-cortical connections of somatic sensory cortex (areas 3, 1 and 2) in the rhesus monkey. J Comp Neurol 177, 179-191.

Wang, Z., Chen, L.M., Negyessy, L., Friedman, R.M., Mishra, A., Gore, J.C., Roe, A.W., 2013. The relationship of anatomical and functional connectivity to resting-state connectivity in primate somatosensory cortex. Neuron $78,1116-$ 1126.

Whitfield-Gabrieli, S., Nieto-Castanon, A., 2012. Conn: a functional connectivity toolbox for correlated and anticorrelated brain networks. Brain Connect 2, 125141. 
Wu, T.L., Mishra, A., Wang, F., Yang, P.F., Gore, J.C., Chen, L.M., 2016. Effects of isoflurane anesthesia on resting-state fMRI signals and functional connectivity within primary somatosensory cortex of monkeys. Brain Behav 6 , e00591.

Yeo, B.T., Krienen, F.M., Sepulcre, J., Sabuncu, M.R., Lashkari, D., Hollinshead, M., Roffman, J.L., Smoller, J.W., Zollei, L., Polimeni, J.R., Fischl, B., Liu, H., Buckner, R.L., 2011. The organization of the human cerebral cortex estimated by intrinsic functional connectivity. J Neurophysiol 106, 1125-1165.

Yousry, T.A., Schmid, U.D., Alkadhi, H., Schmidt, D., Peraud, A., Buettner, A., Winkler, P., 1997. Localization of the motor hand area to a knob on the precentral gyrus. A new landmark. Brain 120 ( Pt 1), 141-157.

Zhou, Z.C., Salzwedel, A.P., Radtke-Schuller, S., Li, Y., Sellers, K.K., Gilmore, J.H., Shih, Y.Y., Frohlich, F., Gao, W., 2016. Resting state network topology of the ferret brain. Neuroimage 143, 70-81. 